This item was submitted to Loughborough's Research Repository by the author.

Items in Figshare are protected by copyright, with all rights reserved, unless otherwise indicated.

\title{
Returns to scale in convex production technologies
}

PLEASE CITE THE PUBLISHED VERSION

http://dx.doi.org/10.1016/j.ejor.2016.09.029

\section{PUBLISHER}

(c) Elsevier

VERSION

AM (Accepted Manuscript)

\section{PUBLISHER STATEMENT}

This work is made available according to the conditions of the Creative Commons Attribution-NonCommercialNoDerivatives 4.0 International (CC BY-NC-ND 4.0) licence. Full details of this licence are available at: https://creativecommons.org/licenses/by-nc-nd/4.0/

\section{LICENCE}

CC BY-NC-ND 4.0

\section{REPOSITORY RECORD}

Podinovski, Victor V.. 2019. "Returns to Scale in Convex Production Technologies". figshare. https://hdl.handle.net/2134/22674. 


\title{
Returns to scale in convex production technologies
}

\author{
Victor V. Podinovski* \\ School of Business and Economics, Loughborough University, Loughborough LE11 3TU, UK
}

\begin{abstract}
The notion of returns to scale (RTS) is well-established in data envelopment analysis (DEA). In the variable returns-to-scale production technology, the RTS characterization is closely related to other scale characteristics, such as the scale elasticity, most productive scale size (MPSS), and global RTS types indicative of the direction to MPSS. In recent years, a number of alternative production technologies have been developed in the DEA literature. Most of these technologies are polyhedral, and hence are closed and convex sets. Examples include technologies with weakly disposable undesirable outputs, models with weight restrictions and production trade-offs, technologies that include several component production processes, and network DEA models. For most of these technologies, the relationship between RTS and other scale characteristics has remained unexplored. The theoretical results obtained in this paper establish such relationships for a very large class of closed convex technologies, of which polyhedral technologies are an important example.
\end{abstract}

Keywords: data envelopment analysis, convex technology, scale elasticity, returns to scale

\section{Introduction}

The notion of returns to scale (RTS) is well-established in data envelopment analysis (DEA) - see, e.g., Cooper et al. (2007), Ray (2004) and Thanassoulis et al. (2008). Extending the earlier results of Banker (1984) and Banker and Thrall (1992), the DEA literature has primarily focused on the definition and evaluation of RTS in the variable returns-to-scale (VRS) production technology, for which several different methods are now available (for a review, see Banker et al., 2011, and Sahoo et al., 2015).

The RTS characterization of decision making units (DMUs) is also related to the notions of scale efficiency and most productive scale size (MPSS) introduced by Banker et al. (1984) and Banker (1984). Further connections can be made to the notion of global RTS (GRS) introduced by Podinovski (2004a, 2004b). The GRS characterization is global in the sense that its types are indicative of the direction to MPSS and are not defined by the local (marginal) properties of production function.

In the VRS technology, some relevant known results describing the relationship between RTS and other scale characteristics can be summarized as follows.

1. A standard procedure for testing if a DMU is at MPSS arises from the definition of MPSS by Banker (1984). It is based on evaluation of input or output radial efficiency of the DMU in the reference constant returns-to-scale (CRS) technology of Charnes et al. (1978), which, from a general perspective, is the cone technology generated by the VRS technology.

*Corresponding author

Email address: v.podinovski@lboro.ac.uk (Victor V. Podinovski) 
2. An alternative way to test for MPSS is to evaluate the type of RTS exhibited by a DMU. Namely, a DMU is at MPSS if and only if it exhibits CRS (Banker and Thrall, 1992).

3. The GRS characterization of DMUs in the VRS technology, while generally different from the conventional local RTS characterization, in the case of VRS technology coincides with the latter. This effectively follows from Proposition 1 proved by Banker (1984).

In recent years, a number of new production technologies have been developed and studied in the DEA literature. Most of these technologies are polyhedral (and therefore convex) sets in the input and output dimensions. Podinovski et al. (2016) refer to such technologies as polyhedral technologies. ${ }^{1}$

The class of polyhedral technologies is very large and includes most of the known convex DEA technologies, such as the CRS and VRS technologies of Charnes et al. (1978) and Banker et al. (1984). Further examples include the VRS and CRS technologies expanded by weight restrictions or production trade-offs (Podinovski, 2004d, 2007, 2015, 2016; Atici and Podinovski, 2015; Podinovski and Bouzdine-Chameeva, 2013, 2015; Joro and Korhonen, 2015), the weakly disposable VRS technology (Kuosmanen, 2005; Kuosmanen and Podinovski, 2009, Kuosmanen and Kazemi Matin, 2011), the hybrid returns-to-scale (HRS) technology (Podinovski, 2004c; Podinovski et al., 2014), the convex CRS technology with exogenously fixed inputs and outputs (Podinovski and Bouzdine-Chameeva, 2011), some models of technologies with multiple component processes (Cherchye et al. 2013; Cherchye et al. 2015, 2016; Cook and Zhu, 2011) and various network DEA models (see, e.g., Kao, 2014 and Sahoo et al., 2014).

It is clear that RTS and related scale characterizations such as MPSS are important for all polyhedral technologies. Thus, several authors develop bespoke methodologies for evaluation of RTS in particular technologies (see, e.g., Tone, 2001 and Sahoo et al., 2014). Podinovski et al. (2016) develop a universal methodology for the RTS characterization of DMUs in any polyhedral technology. This approach uses linear programming techniques for calculation of one-sided scale elasticities that subsequently define the types of RTS.

Although the current DEA literature allows us to define and evaluate the RTS types for any polyhedral technology, and further methods exist for their GRS characterization, the relationship between RTS and GRS types (including MPSS) has so far remained unexplored. An exception here is the equivalence of RTS and GRS characterizations for convex technologies whose boundaries are smooth, established by Podinovski (2004a). This result does not, however, apply to polyhedral technologies.

This paper addresses the above gap. Its main contribution is the establishment of equivalence of local and global characterizations of RTS in any polyhedral technology. In particular, this implies that a DMU exhibits CRS if and only if it is at MPSS. In fact, from the theoretical perspective, it is straightforward to generalize and prove this result in a larger class of closed convex technologies, of which polyhedral technologies are a special case.

From a practical perspective, the established equivalence of the notions of RTS and GRS gives us a new tool for evaluating the GRS types in any polyhedral (and, more generally, closed and convex) technology, by evaluating the RTS types instead. More precisely, standard methods for the evaluation of MPSS and GRS types require the use of reference technologies (such as the CRS, non-increasing and non-decreasing RTS technologies, if the

\footnotetext{
${ }^{1}$ In a finite-dimensional space $\mathbb{R}^{n}$ a polyhedral set is defined as the intersection of a finite number of closed half-spaces (Rockafellar, 1970).
} 
underlying true technology is VRS). For many polyhedral technologies, their reference technologies may not be immediately available and would require further development before they could be used. The new theoretical results established in this paper allow us to avoid this and, instead, use the existing methodologies for evaluation of RTS.

We illustrate the usefulness of the new results by a numerical example involving the RTS and GRS characterizations of a VRS technology expanded by the specification of weight restrictions. We also discuss the application of new results to a two-stage network DEA model.

\section{The output response function}

Consider a production technology $\mathcal{T} \in \mathbb{R}_{+}^{m+s}$, where $m$ is the number of inputs and $s$ is the number of outputs. ${ }^{2}$ Elements of $\mathcal{T}$ are DMUs $(X, Y)$, where $X \in \mathbb{R}_{+}^{m}$ and $Y \in \mathbb{R}_{+}^{s}$ are the vectors of inputs and outputs, respectively.

Unless stated otherwise, the only two general assumptions we make about $\mathcal{T}$ is that it is a closed and convex set. ${ }^{3}$ We refer to $\mathcal{T}$ as a closed convex technology. As noted above, a practically important example of such technologies is the class of polyhedral technologies.

Consider any $\operatorname{DMU}\left(X_{o}, Y_{o}\right) \in \mathcal{T}$, where $\mathcal{T}$ is a closed convex technology. Throughout this paper we assume that $X_{o} \neq \varnothing$ and $Y_{o} \neq \varnothing$. Central to our development is the output response function $\bar{\beta}(\alpha)$ defined as follows:

$$
\bar{\beta}(\alpha)=\max \left\{\beta \mid\left(\alpha X_{o}, \beta Y_{o}\right) \in \mathcal{T}, \beta \in \mathbb{R}\right\} .
$$

In formula (1), the scalar $\alpha$ defines a proportional change to the input vector $X_{o}$, and $\beta$ defines a proportional change to the output vector $Y_{o}$. The output response function $\bar{\beta}(\alpha)$ is equal to the maximum proportion $\beta$ of output vector $Y_{o}$ that can be produced in technology $\mathcal{T}$ from the input vector $\alpha X_{o}{ }^{4}$

We also make the following assumption:

Assumption 1. DMU $\left(X_{o}, Y_{o}\right)$ is output radial efficient, i.e.,

$$
\bar{\beta}(1)=\max \left\{\beta \mid\left(X_{o}, \beta Y_{o}\right) \in \mathcal{T}, \beta \in \mathbb{R}\right\}=1 .
$$

Let $\Gamma$ be the domain of function $\bar{\beta}(\alpha)$, i.e., $\Gamma \in \mathbb{R}$ is the set of all $\alpha$ for which there exists a $\beta$ such that $\left(\alpha X_{o}, \beta Y_{o}\right) \in \mathcal{T}$. Clearly, $1 \in \Gamma$.

Proposition 1. (i) $\Gamma$ is a closed interval in $\mathbb{R}_{+}$;

(ii) For all $\alpha \in \Gamma$, the maximum in (1) is finite and is attained.

(iii) $\bar{\beta}(\alpha)$ is a continuous and concave function on $\Gamma$.

\footnotetext{
${ }^{2}$ The requirement that $\mathcal{T}$ is a subset of the nonnegative orthant $\mathbb{R}_{+}^{m+s}$ is needed for the correct definition of RTS types and MPSS. However, this requirement is not needed and is omitted for the definition and calculation of the scale elasticity and other marginal scale characteristics. In the case of polyhedral technologies this is demonstrated by Podinovski et al. (2016).

${ }^{3}$ A further Assumption 1 additionally implies that technology $\mathcal{T}$ does not allow unlimited production. (This follows from Theorem 2 proved in Podinovski and Bouzdine-Chameeva 2013.) Technology $\mathcal{T}$ allows unlimited production if there exists an input vector $X^{*}$ and output vector $Y^{*} \neq 0$ such that $\sup \left\{\beta \mid\left(X^{*}, \beta Y^{*}\right) \in \mathcal{T}\right\}=+\infty$.

${ }^{4}$ The function $\bar{\beta}(\alpha)$ is closely related to the directional distance function of Chambers et al. (1998) assessed at DMU $\left(X_{o}, Y_{o}\right)$ in the direction of vector $Y_{o}$. For details of this correspondence, see, e.g., Section 3.2 in Podinovski et al. (2016).
} 
The proofs of Proposition 1 and the other statements are given in Appendix A.

The RTS characterization of DMUs developed below is based on the notion of right-hand and left-hand derivatives of the function $\bar{\beta}(\alpha)$, denoted $\bar{\beta}_{+}^{\prime}(\alpha)$ and $\bar{\beta}_{-}^{\prime}(\alpha)$, respectively. As follows from Theorem 24.1 in Rockafellar (1970) restated for a concave function $\bar{\beta}(\alpha)$, we have the following properties of the one-sided derivatives $\bar{\beta}_{+}^{\prime}(\alpha)$ and $\bar{\beta}_{-}^{\prime}(\alpha)$ taking values from the interval $[-\infty,+\infty]$ :

1. The one-sided derivatives $\bar{\beta}_{+}^{\prime}(\alpha)$ and $\bar{\beta}_{-}^{\prime}(\alpha)$ exist for all $\alpha \in \Gamma$. For any $\alpha$ interior to $\Gamma$, both $\bar{\beta}_{+}^{\prime}(\alpha)$ and $\bar{\beta}_{-}^{\prime}(\alpha)$ are finite.

2. If $\alpha$ is the left extreme point of $\Gamma, \bar{\beta}_{+}^{\prime}(\alpha)$ may be finite or $+\infty$. Similarly, if $\alpha$ is the right extreme point of $\Gamma, \bar{\beta}_{-}^{\prime}(\alpha)$ may be finite or $-\infty .^{5}$

3. For all $\alpha_{1}, \alpha_{2}, \alpha_{3} \in \Gamma$ such that $\alpha_{1}<\alpha_{2}<\alpha_{3}$, we have the following monotonicity property:

$$
\bar{\beta}_{+}^{\prime}\left(\alpha_{1}\right) \geq \bar{\beta}_{-}^{\prime}\left(\alpha_{2}\right) \geq \bar{\beta}_{+}^{\prime}\left(\alpha_{2}\right) \geq \bar{\beta}_{-}^{\prime}\left(\alpha_{3}\right) .
$$

For convenience, we also formally define $\bar{\beta}_{-}^{\prime}(\alpha)=+\infty$ if $\alpha$ is the left extreme point of $\Gamma$, and $\bar{\beta}_{+}^{\prime}(\alpha)=-\infty$ if $\alpha$ is the right extreme point of $\Gamma$. Although neither of these one-sided derivatives exists in the classical sense, this definition helps us to avoid giving a special consideration of the extreme points of $\Gamma$ in the definition of RTS below.

Remark 1. If technology $\mathcal{T}$ is polyhedral, in addition to the properties stated in Proposition 1 , the function $\bar{\beta}(\alpha)$ is also piecewise linear on $\Gamma{ }^{6}$ This in turn implies that, if $\alpha$ is the left extreme point of $\Gamma, \bar{\beta}_{+}^{\prime}(\alpha)$ is finite. Similarly, if $\alpha$ is the right extreme point of $\Gamma, \bar{\beta}_{-}^{\prime}(\alpha)$ is finite.

As becomes clear below, of particular importance to us are the one-sided derivatives evaluated at $\alpha=1$. For any polyhedral technology, both derivatives can be calculated using the linear programs developed by Podinovski et al. (2016).

\section{The one-sided scale elasticity}

If $\alpha=1$ is an interior point of domain $\Gamma$ and the function $\bar{\beta}(\alpha)$ is differentiable at $\alpha=1$, the scale elasticity $\varepsilon\left(X_{o}, Y_{o}\right)$ evaluated at DMU $\left(X_{o}, Y_{o}\right)$ can be defined as

$$
\varepsilon\left(X_{o}, Y_{o}\right)=\bar{\beta}^{\prime}(1)
$$

The meaning of this definition is straightforward. To be specific, let $\varepsilon\left(X_{o}, Y_{o}\right)=2$. Suppose we increase the input vector $X_{o}$ in a small proportion, e.g., by $1 \%$ (corresponding to $\alpha=1.01)$. Then the maximum proportion of vector $Y_{o}$ possible in technology $\mathcal{T}$ increases (to the first degree of approximation) by $\varepsilon\left(X_{o}, Y_{o}\right)=2 \%$ (which corresponds to $\beta=1.02$ ). ${ }^{7}$ Similarly, if we reduce the components of vector $X_{o}$ by $1 \%$, the maximum proportion of vector $Y_{o}$ is reduced by $\varepsilon\left(X_{o}, Y_{o}\right)=2 \%(\alpha=0.99$ and $\beta=0.98)$.

Podinovski and Førsund (2010) prove that the above definition of scale elasticity $\varepsilon\left(X_{o}, Y_{o}\right)$, effectively also used by Banker (1984) and Banker and Thrall (1992), is equivalent to its standard definition given in terms of partial derivatives of the production function $\Phi(X, Y)$

\footnotetext{
${ }^{5}$ For example, consider the closed convex technology $\mathcal{T}$ with a single input and output that contains all DMUs located under the curve $Y=1+(X-1)^{1 / 2}$, where $X \geq 1, Y \geq 0$. Then DMU $\left(X_{o}, Y_{o}\right)=(1,1)$ satisfies Assumption 1, and we have $\bar{\beta}_{+}^{\prime}(1)=+\infty$.

${ }^{6}$ This follows from Proposition 2 stated in Podinovski et al. (2016), which is a restatement of a known result of sensitivity analysis in linear optimization.

${ }^{7}$ The corresponding difference quotent is equal to $(\bar{\beta}(\alpha)-\bar{\beta}(1)) /(\alpha-1)=(1.02-1) /(1.01-1)=2$.
} 
(Hanoch, 1970; Panzar and Willig, 1977) ${ }^{8}$ and, provided $\mathcal{T}$ is freely disposable in all outputs, to its definition via the notion of local degrees of homogeneity $h$ (Panzar and Willig, 1977; Starrett, 1977).

Clearly, for general convex production technologies, the function $\bar{\beta}(\alpha)$ cannot be assumed differentiable, and the standard economic notion of scale elasticity (3) is undefined. In particular, this definition does not apply to the VRS technology. This problem is overcome by the introduction of one-sided (right-hand and left-hand) scale elasticities $\varepsilon^{+}\left(X_{o}, Y_{o}\right)$ and $\varepsilon^{-}\left(X_{o}, Y_{o}\right)$ evaluated at $\mathrm{DMU}_{o}$, which correspond to the one-sided derivatives $\bar{\beta}_{+}^{\prime}(1)$ and $\bar{\beta}_{-}^{\prime}(1)$.

For the conventional VRS technology this approach was pioneered by Banker and Thrall (1992) and further explored by Fukuyama (2000), Hadjicostas and Soteriou (2006), Podinovski et al. (2009), Podinovski and Førsund (2010), and Zelenyuk (2013). This approach is extended to the entire class of polyhedral technologies by Podinovski et al. (2016). ${ }^{9}$

Because, as shown above, the one-sided derivatives of function $\bar{\beta}(\alpha)$ are well-defined in the more general case of closed production technologies, the definition of one-sided scale elasticities based on them is straightforward.

Let $\mathcal{T}$ be a closed convex technology, and let DMU $\left(X_{o}, Y_{o}\right) \in \mathcal{T}$ satisfy Assumption 1 .

Definition 1. The one-sided scale elasticities at DMU $\left(X_{o}, Y_{o}\right)$ are defined as follows:

$$
\begin{aligned}
& \varepsilon^{+}\left(X_{o}, Y_{o}\right)=\bar{\beta}_{+}^{\prime}(1), \\
& \varepsilon^{-}\left(X_{o}, Y_{o}\right)=\bar{\beta}_{-}^{\prime}(1) .
\end{aligned}
$$

Note that the middle inequality in (2) implies

$$
\varepsilon^{+}\left(X_{o}, Y_{o}\right) \leq \varepsilon^{-}\left(X_{o}, Y_{o}\right) .
$$

If $\varepsilon^{+}\left(X_{o}, Y_{o}\right)=\varepsilon^{-}\left(X_{o}, Y_{o}\right)$ (or, equivalently, $\bar{\beta}_{+}^{\prime}(1)=\bar{\beta}_{-}^{\prime}(1)$, and the function $\bar{\beta}(\alpha)$ is therefore differentiable at $\alpha=1)$, we have $\varepsilon\left(X_{o}, Y_{o}\right)=\varepsilon^{+}\left(X_{o}, Y_{o}\right)=\varepsilon^{-}\left(X_{o}, Y_{o}\right)$.

If $\varepsilon^{+}\left(X_{o}, Y_{o}\right)<\varepsilon^{-}\left(X_{o}, Y_{o}\right)$, the scale elasticity (elasticity of response of output vector $Y_{o}$ to marginal changes of input vector $X_{o}$ ) is different if vector $X_{o}$ is proportionally increased or reduced. For example, let $\varepsilon^{+}\left(X_{o}, Y_{o}\right)=0.5$ and $\varepsilon^{-}\left(X_{o}, Y_{o}\right)=1.5$. Then, if we increase the input vector $X_{o}$ proportionally by $1 \%$, the maximum possible proportion of vector $Y_{o}$ increases by $\varepsilon^{+}\left(X_{o}, Y_{o}\right)=0.5 \%$. On the other hand, if we reduce vector $X_{o}$ by $1 \%$, the maximum proportion of vector $Y_{o}$ is reduced by $\varepsilon^{-}\left(X_{o}, Y_{o}\right)=1.5 \%$.

Remark 2. There exist two equivalent methods suitable for the calculation of one-sided scale elasticities at any $\operatorname{DMU}\left(X_{o}, Y_{o}\right)$. First, as noted in Remark 1 , if technology $\mathcal{T}$ is polyhedral, the one-sided derivatives $\bar{\beta}_{+}^{\prime}(1)$ and $\bar{\beta}_{-}^{\prime}(1)$, and therefore the one-sided scale elasticities $\varepsilon^{+}\left(X_{o}, Y_{o}\right)$ and $\varepsilon^{-}\left(X_{o}, Y_{o}\right)$, can be computed using the linear programming approach of Podinovski et al. (2016).

Second, as is well-known from the literature, if $\mathcal{T}$ is the VRS technology of Banker et al. (1984), these one-sided elasticities can equivalently be defined as follows:

$$
\begin{aligned}
& \varepsilon^{+}\left(X_{o}, Y_{o}\right)=1-\omega^{\max }, \\
& \varepsilon^{-}\left(X_{o}, Y_{o}\right)=1-\omega^{\min },
\end{aligned}
$$

\footnotetext{
${ }^{8}$ The notion of production function $\Phi(X, Y)$ is used to give an implicit definition of the production frontier by the equation $\Phi(X, Y)=0$. Such a definition requires that the function $\Phi(X, Y)$ satisfies certain properties, e.g., the monotonicity in $X$ and $Y$ (Hanoch, 1970; Panzar and Willig, 1977).

${ }^{9}$ Theoretical foundations of this approach are explored by Chambers and Färe (2008). In a further extension, Podinovski and Førsund (2010), Atici and Podinovski (2012) and Podinovski et al. (2016) consider partial one-sided scale elasticities that correspond to the case in which a subset of input and output measures responds to marginal changes of another subset.
} 
where $\omega^{\text {max }}$ and $\omega^{\text {min }}$ are, respectively, the maximum and minimum of the sign free variable $\omega$ dual to the normalizing equality $1^{\top} \lambda=1$, taken over the set of optimal solutions to the output-oriented multiplier VRS model (Førsund and Hjalmarsson, 2004). A similar formula to (4) can be given in terms of variable $\omega$ calculated in the input-oriented VRS model (Førsund and Hjalmarsson, 2004; Podinovski et al., 2009; Zelenyuk, 2013). Podinovski et al. (2016) extend formula (4) and its analogue based on the input-oriented model, to any polyhedral technology. We use formula (4) for the calculation of one-sided scale elasticities in the example in Section 8.

\section{Returns to scale}

According to the standard definition (see, e.g., Førsund and Hjalmarsson 2004), the type of RTS exhibited by an output efficient DMU $\left(X_{o}, Y_{o}\right)$ is defined by the scale elasticity assessed at this DMU. In particular, DMU $\left(X_{o}, Y_{o}\right)$ exhibits increasing, decreasing or constant RTS (IRS, DRS or CRS) if $\varepsilon\left(X_{o}, Y_{o}\right)>1, \varepsilon\left(X_{o}, Y_{o}\right)<1$, or $\varepsilon\left(X_{o}, Y_{o}\right)=1$, respectively.

Banker and Thrall (1992) generalize this definition to the VRS technology by utilizing the one-sided scale elasticities (see also Banker et al., 2011). Podinovski et al. (2016) extend the approach of Banker and Thrall (1992) to all polyhedral technologies. Its further extension to any closed convex technology is also straightforward.

Let, as above, $\mathcal{T}$ be a closed convex technology, and let DMU $\left(X_{o}, Y_{o}\right) \in \mathcal{T}$ satisfy Assumption $1 .^{10}$

Definition 2. DMU $\left(X_{o}, Y_{o}\right)$ exhibits

(i) IRS if $1<\varepsilon^{+}\left(X_{o}, Y_{o}\right) \leq \varepsilon^{-}\left(X_{o}, Y_{o}\right)$;

(ii) DRS if $\varepsilon^{+}\left(X_{o}, Y_{o}\right) \leq \varepsilon^{-}\left(X_{o}, Y_{o}\right)<1$;

(iii) CRS if $\varepsilon^{+}\left(X_{o}, Y_{o}\right) \leq 1 \leq \varepsilon^{-}\left(X_{o}, Y_{o}\right)$.

Several remarks with respect to the above definition are worth making. First, the IRS and DRS types are conceptually consistent with the standard definition of RTS that applies if the scale elasticity $\varepsilon\left(X_{o}, Y_{o}\right)$ exists. Consider, for example the case of IRS in Definition 2. Although the elasticity of response of output vector $Y_{o}$ to marginal changes of input vector $X_{o}$ may generally be different in the case of vector $X_{o}$ increasing or decreasing, both measures $\varepsilon^{+}\left(X_{o}, Y_{o}\right)$ and $\varepsilon^{-}\left(X_{o}, Y_{o}\right)$ are assumed to be greater than 1. Therefore, in both cases the proportional change (positive or negative) to vector $Y_{o}$ is greater than the change to vector $X_{o}$. Similarly, in the case of DRS, the proportional change to vector $Y_{o}$ described by $\varepsilon^{+}\left(X_{o}, Y_{o}\right)$ and $\varepsilon^{-}\left(X_{o}, Y_{o}\right)$ is less than the change to vector $X_{o}$.

Second, in the case of CRS in Definition 2, we effectively have DRS if vector $X_{o}$ is marginally increased, and IRS if $X_{o}$ is reduced. This is of course not the standard notion of CRS that requires that the scale elasticity (and hence its both one-sided analogues) be equal to 1, but such a classification appears to be well-established in the literature (see, e.g., Banker and Thrall, 1992; Banker et al., 2011). Furthermore, as we prove below, in this case

\footnotetext{
${ }^{10}$ In the DEA literature, the RTS characterization is often limited to fully efficient DMUs. However, there is no real reason for this as a weaker Assumption 1 of output radial efficiency of $\mathrm{DMU}_{o}$ suffices for the correct definition of RTS (Podinovski and Førsund, 2010; Podinovski et al., 2016). The notion of RTS is sometimes also extended to DMUs that are not output radial efficient. This requires that $\mathrm{DMU}_{o}$ is first projected on the boundary of the technology, e.g., by means of output radial maximization or input minimization. Provided the target DMU satisfies Assumption 1 (which is always true for the output radial projections but is not necessarily true for the input projections), the type of RTS exhibited by the target DMU is assigned to the inefficient $\mathrm{DMU}_{o}$. It is well-known that different projections of the same inefficient DMU may result in its different RTS characterizations (see, e.g., Banker et al., 2011).
} 
$\operatorname{DMU}\left(X_{o}, Y_{o}\right)$ attains the maximum productivity on the domain $\Gamma$, i.e., is at MPSS. The latter is consistent with the standard CRS type.

Third, suppose that vector $X_{o}$ cannot be proportionally reduced in technology $\mathcal{T}$. (For example, this situation often arises in computations of scale elasticity in the VRS technology.) In this case $\alpha=1$ is the left extreme point of the interval $\Gamma$, and the left-hand derivative $\bar{\beta}_{-}^{\prime}(1)$ is undefined. As noted above, in this case we formally define $\bar{\beta}_{-}^{\prime}(1)=+\infty$, and so $\varepsilon^{-}\left(X_{o}, Y_{o}\right)=+\infty$. According to Definition 2, this means that any DMU $\left(X_{o}, Y_{o}\right)$ whose vector $X_{o}$ cannot be proportionally reduced in $\mathcal{T}$, cannot exhibit DRS. Moreover, whether DMU $\left(X_{o}, Y_{o}\right)$ is classed as exhibiting IRS or CRS depends entirely on the right-hand scale elasticity $\varepsilon^{+}\left(X_{o}, Y_{o}\right)$. A similar observation applies if vector $X_{o}$ cannot be proportionally increased in $\mathcal{T}$ (although this possibility is more of theoretical interest as it cannot arise if $\mathcal{T}$ is freely disposable in all inputs, which is normally assumed).

Remark 3. As follows from Remark 2, if $\mathcal{T}$ is the VRS technology, Definition 2 can be restated in terms of the maximum and minimum values $\omega^{\max }$ and $\omega^{\min }$ of variable $\omega$ in the output-oriented VRS model using formulae (4), and also in terms of $\omega$ evaluated in the input-oriented model (see, e.g., Sahoo and Tone, 2015). A similar restatement is possible if $\mathcal{T}$ is a general polyhedral technology (Podinovski et al., 2016).

Remark 4. It is intuitively clear that the shape of production frontier and the (one-sided) scale elasticities that characterize its different parts are generally sensitive to the set of observed DMUs on which the analysis is based. For the standard VRS technology of Banker et al. (1984), Podinovski and Førsund (2010) prove that, if more DMUs are added to the sample and $\mathrm{DMU}_{o}$ remains output radial efficient in the new (larger) VRS technology, then the left-hand scale elasticity $\varepsilon^{-}\left(X_{o}, Y_{o}\right)$ cannot increase, while the right-hand scale elasticity $\varepsilon^{+}\left(X_{o}, Y_{o}\right)$ cannot decrease. This implies that the interval $\left[\varepsilon^{+}\left(X_{o}, Y_{o}\right), \varepsilon^{-}\left(X_{o}, Y_{o}\right)\right]$ generally becomes narrower as more DMUs are added to the sample. By Definition 2 this means that a $\mathrm{DMU}_{o}$ classed as exhibiting IRS will remain in the same class if the sample is enlarged (provided $\mathrm{DMU}_{o}$ still satisfies Assumption 1 in the enlarged VRS technology). Similarly, a $\mathrm{DMU}_{o}$ exhibiting DRS, will exhibit DRS in a larger sample. However, a $\mathrm{DMU}_{o}$ exhibiting CRS may exhibit any of the three types of RTS in the enlarged sample.

\section{Most productive scale size}

Consider any (not necessarily convex) production technology $\mathcal{T}$. According to the definition given by Banker (1984), a DMU $\left(X_{o}, Y_{o}\right) \in \mathcal{T}$ is at MPSS if for all DMUs in the form $\left(\alpha X_{o}, \beta Y_{o}\right) \in \mathcal{T}$, where $\alpha>0$, we have $\beta / \alpha \leq 1{ }^{11}$ Therefore, following Banker (1984), $\operatorname{DMU}\left(X_{o}, Y_{o}\right)$ is at MPSS if the optimal value of the following program is equal to 1 :

$$
\begin{array}{cl}
\max & \beta / \alpha \\
\text { s.t. } & \left(\alpha X_{o}, \beta Y_{o}\right) \in \mathcal{T}, \\
& \alpha, \beta>0 .
\end{array}
$$

Obviously, if DMU $\left(X_{o}, Y_{o}\right)$ is at MPSS, it is output radial efficient and therefore satisfies Assumption 1.

Program (5) can be simplified by introducing the reference cone extension $\mathcal{T}^{*}$ to technology $\mathcal{T}$ :

$$
\mathcal{T}^{*}=\left\{(X, Y) \in \mathbb{R}^{m+s} \mid \exists(\tilde{X}, \tilde{Y}) \in \mathcal{T}, \delta \geq 0:(X, Y)=\delta(\tilde{X}, \tilde{Y})\right\}
$$

\footnotetext{
${ }^{11}$ This standard definition is equivalently restated using the output response function $\bar{\beta}(\alpha)$. Namely, DMU $\left(X_{o}, Y_{o}\right)$ is at MPSS if $\bar{\beta}(\alpha) / \alpha \leq 1$, for all $\alpha \in \Gamma \backslash\{0\}$.
} 
Note that the optimal value of program (5) does not change if we replace $\mathcal{T}$ by $\mathcal{T}^{*}$ in its constraints. ${ }^{12}$ We can subsequently normalize the feasible solutions by requiring that $\alpha=1$ and remove the nonnegativity conditions on variables $\alpha$ and $\beta$ as redundant. ${ }^{13}$ Therefore, the optimal value of program (5) is equal to the optimal value of the following program:

$$
\begin{aligned}
\max & \beta \\
\text { s.t. } & \left(X_{o}, \beta Y_{o}\right) \in \mathcal{T}^{*} .
\end{aligned}
$$

Program (6) measures the output radial efficiency of DMU $\left(X_{o}, Y_{o}\right)$ in technology $\mathcal{T}^{*}$. Therefore, DMU $\left(X_{o}, Y_{o}\right)$ is at MPSS if and only if it is output radial efficient in the cone technology $\mathcal{T}^{*}$ generated by $\mathcal{T}$. If $\mathcal{T}$ is the VRS technology of Banker et al. (1984), program (6) is the standard output-oriented CRS model of Charnes et al (1978).

\section{Global returns to scale}

The notion of global returns to scale (GRS) was introduced by Podinovski (2004a, 2004b) under extremely weak assumptions about the production technology $\mathcal{T}$, namely, that the latter does not allow free and unlimited production. ${ }^{14}$ No other assumptions about $\mathcal{T}$ are required. For example, $\mathcal{T}$ is not required to be convex, disposable according to any definition or even closed.

The types of GRS are indicative of the direction in which the size of operations of $\mathrm{DMU}_{o}$ should change while approaching its MPSS. To state this formally and following Podinovski (2004a), let $\mathcal{T} \in \mathbb{R}_{+}^{m+s}$ be any technology that disallows free and unlimited production, and let $\operatorname{DMU}\left(X_{o}, Y_{o}\right) \in \mathcal{T}$ satisfy Assumption 1 .

Suppose that the optimal value of program (5) is attained at some $\hat{\alpha}$ and $\hat{\beta}$. Obviously, $\operatorname{DMU}\left(\hat{\alpha} X_{o}, \hat{\beta} Y_{o}\right)$ is at MPSS. Podinovski $(2004 \mathrm{a}, 2004 \mathrm{~b})$ refers to this DMU as the scale reference unit $(\mathrm{SRU})$ of DMU $\left(X_{o}, Y_{o}\right)$.

If $\operatorname{DMU}\left(X_{o}, Y_{o}\right)$ is at MPSS, then $\hat{\alpha}=\hat{\beta}=1$ is an optimal solution to (5), and $\left(\hat{\alpha} X_{o}, \hat{\beta} Y_{o}\right)=\left(X_{o}, Y_{o}\right)$. Otherwise, $\mathrm{DMU}_{o}$ is either smaller than its $\operatorname{SRU}\left(\hat{\alpha} X_{o}, \hat{\beta} Y_{o}\right)$ (if $\hat{\alpha}, \hat{\beta}>1$ ), or $\mathrm{DMU}_{o}$ is larger than its $\mathrm{SRU}$ (if $\left.\hat{\alpha}, \hat{\beta}<1\right) .{ }^{15}$ It is also possible that program (5) has multiple optimal solutions, each defining a different $\mathrm{SRU}$ of $\mathrm{DMU}_{o}$. In this case it is theoretically possible that $\mathrm{DMU}_{o}$ is smaller than some of its SRUs and larger than the other. ${ }^{16}$

Definition 3. (Podinovski, 2004a). DMU $\left(X_{o}, Y_{o}\right)$ exhibits

(i) global CRS (G-CRS) if $\mathrm{DMU}_{o}$ is at MPSS;

\footnotetext{
${ }^{12}$ Any feasible solution in the resulting program (with $\mathcal{T}$ replaced by $\mathcal{T}^{*}$ ) can be stated as $(\delta \alpha, \delta \beta)$, where $(\alpha, \beta)$ is feasible in (5). Because $(\delta \beta) /(\delta \alpha)=\beta / \alpha, \forall \alpha, \delta>0$, the feasible values of the objective function in (5) are not affected.

${ }^{13}$ Let $\left(\alpha^{*}, \beta^{*}\right)$ be an optimal solution to program (5) in which $\mathcal{T}$ is replaced by $\mathcal{T}^{*}$. Then $\left(\delta \alpha^{*}, \delta \beta^{*}\right)$ is also an optimal solution, for any $\delta>0$. By taking $\delta=1 / \alpha^{*}$, we obtain the optimal solution $\left(1, \beta^{*} / \alpha^{*}\right)$. Therefore, there always exists an optimal solution in the form $(1, \beta)$. Program (6) identifies such a solution.

${ }^{14}$ Technology $\mathcal{T}$ allows free production if there exists a $\operatorname{DMU}\left(X^{*}, Y^{*}\right) \in \mathcal{T}$ such that $X^{*}=0$ and $Y^{*} \neq 0$. The notion of unlimited production was defined in footnote 3 .

${ }^{15}$ By Lemma 2 in Podinovski (2004a), $\hat{\alpha}<1$ implies $\hat{\beta}<1$, and $\hat{\alpha}>1$ implies $\hat{\beta}>1$, so, for example, the case $\hat{\alpha}<1$ and $\hat{\beta}>1$ is impossible.

${ }^{16}$ Let $\beta^{*}$ be the optimal value of program (6). Then the condition $\left(\alpha X_{o}, \alpha \beta^{*} Y_{o}\right) \in \mathcal{T}$, where $\alpha>0$, defines all SRUs of $\mathrm{DMU}_{o}$. The smallest SRU is found by minimizing $\alpha$ subject to the latter condition, and the largest SRU is identified by maximizing $\alpha$ subject to the same condition. If $\mathcal{T}$ is a polyhedral technology, each of the latter two tasks requires solving an appropriately specified linear program.
} 
(ii) global IRS (G-IRS) if all its SRUs are larger than $\mathrm{DMU}_{o}$;

(iii) global DRS (G-DRS) if all its SRUs are smaller than $\mathrm{DMU}_{o}$;

(iv) global sub-constant CRS (G-SCRS) if some of its SRUs are smaller, and some larger, than $\mathrm{DMU}_{o}$, but $\mathrm{DMU}_{o}$ is not at MPSS. ${ }^{17}$

In the general case program (5) may not have an optimal solution and its maximum (in this case more correctly referred to as the supremum) may not be attained. Consequently, an SRU of $\mathrm{DMU}_{o}$ does not exist. In this case Podinovski (2004a) introduces an approximate notion of SRU referred to $\varepsilon$-SRU, and restates Definition 3 in the latter terms.

Podinovski (2004a) shows that the type of GRS exhibited by $\mathrm{DMU}_{o}$ can be tested by evaluating its output radial efficiency in technology $\mathcal{T}$ and two further reference technologies, namely, in the nonincreasing (NIRS) and nondecreasing (NDRS) returns-to-scale technologies generated by $\mathcal{T}{ }^{18}$ The four types of GRS correspond to the four logical possibilities arising by comparison of the efficiencies of $\mathrm{DMU}_{o}$ in the three technologies. ${ }^{19}$

It is worth noting that the local and global characterizations of RTS are conceptually different. The local characterization of RTS is based on the notion of scale elasticity (which can be generalized using its one-sided analogues). Provided the efficient frontier is sufficiently smooth, the scale elasticity is a particular derivative evaluated at $\mathrm{DMU}_{o}$ (i.e., $\bar{\beta}^{\prime}(1)$ in our notation). This derivative depends only on a marginally small neighbourhood of $\mathrm{DMU}_{o}$ or, equivalently, on a marginally small neighbourhood of $\alpha=1$ within the domain $\Gamma$. Therefore, the types of RTS are indicative of the direction that $\mathrm{DMU}_{o}$ should undertake for immediate marginal improvements of its productivity. For example, if $\mathrm{DMU}_{o}$ exhibits IRS, its productivity will increase if $\mathrm{DMU}_{o}$ increases the scale of its operations in a small proportion.

In contrast, the GRS types are global characteristics. They indicate a direction that $\mathrm{DMU}_{o}$ should undertake as it changes the scale of its operations towards its MPSS, even if moving in the identified direction in small steps might initially lead to a detriment to its productivity.

For example, consider the nonconvex technology with a single input $X$ and single output $Y$ shown as the shaded area in Figure 1. Note that DMUs $A, C$ and $E$ exhibit local CRS, DMU $B$ exhibits local DRS, and DMU $D$ exhibits local IRS. From the global perspective, DMU $A$ has the largest ratio of the output to the input, and is the only DMU that is at MPSS. Therefore, DMU $A$ exhibits G-CRS. The other three DMUs $B, C, D$ and $E$ are larger than $A$ and, therefore, all exhibit G-DRS.

\footnotetext{
${ }^{17}$ The G-SCRS type is primarily needed for the theoretical completeness of the GRS characterization, and may be observed in specially constructed illustrative examples (Podinovski, 2004a, 2004b). In most practical applications, this type may be rarely observed, and, if technology $\mathcal{T}$ is convex, it is theoretically impossible (Podinovski 2004a). However, in the (nonconvex) free replication hull (FRH) technology of Ray and $\mathrm{Hu}$ (1997) (see also Ray, 2004, p. 144), the G-SCRS type may occur more naturally. For example, consider the FRH generated by two DMUs $A=(1,1)$ and $B=(1.5,1.2)$, where the first component is input and the second is output. Both DMUs are output radial efficient. Note that DMU $A$ and all its replications $k A=(k, k)$, where $k=2,3, \ldots$, are SRUs for DMU $B$. (The ratio of output to input is equal to 1 for DMU $A$ and its replications, and 0.8 for DMU B.) Because DMU $A$ is smaller than $B$, but its replications $k A$, $k=2,3, \ldots$, are larger than $B$, DMU $B$ exhibits G-SCRS.

${ }^{18}$ If $\mathcal{T}$ is free disposal hull $(\mathrm{FDH})$ of Deprins et al. (1984), the output radial efficiency of $\mathrm{DMU}_{o}$ in the corresponding reference technologies can be evaluated by solving mixed integer linear programs developed by Podinovski (2004e).

${ }^{19}$ This method of testing GRS is similar, but not identical, to the reference technology method of testing RTS developed by Färe et al. $(1983,1985)$ and further explored by Kerstens and Vanden Eeckaut (1999) and Briec et al. (2000).
} 


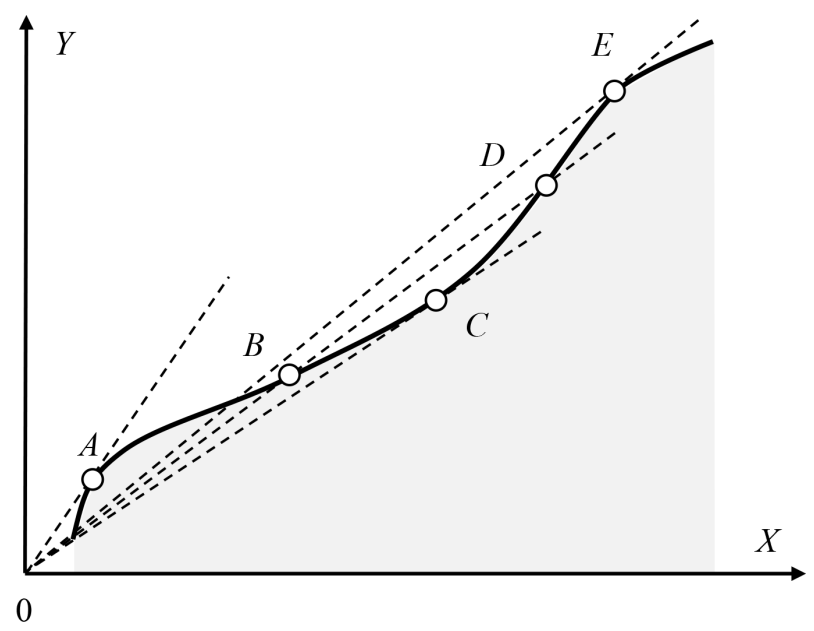

Figure 1: The difference between the local and global RTS characterizations in a nonconvex technology.

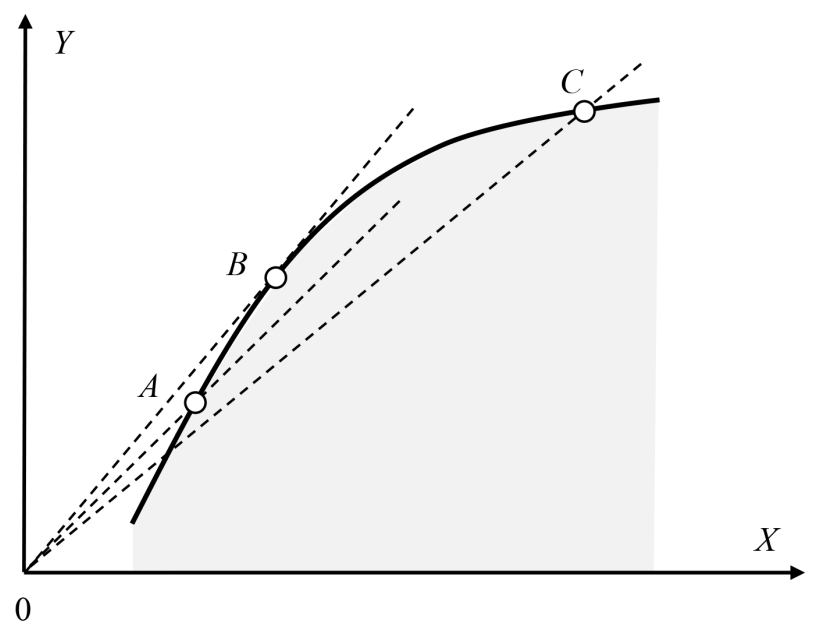

Figure 2: The equivalence of the local and global RTS characterizations in a convex technology.

It is also worth emphasizing that the local characterization of RTS is defined only for sufficiently smooth (convex or nonconvex) production technologies, as required for the definition of scale elasticity or its one-sided analogues. In contrast, the global characterization of GRS does not require that the technology be smooth. For example, the notion of GRS is fully applicable for the characterization of DMUs in free disposal hull (FDH) technology (Deprins et al., 1984).

\section{Equivalence of local and global RTS characterizations}

As noted, in an arbitrary technology, the RTS and GRS characterizations are generally different. However, as proved by Podinovski (2004a, Theorem 7), if technology $\mathcal{T}$ is closed, convex and its frontier is sufficiently smooth, the two characterizations are equivalent. More

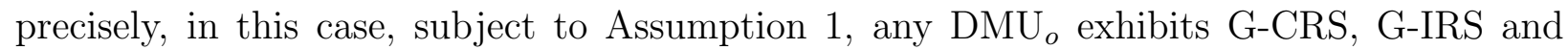
G-DRS if and only if it exhibits local CRS, IRS and DRS, respectively. In this case, the G-SCRS type is impossible.

For example, consider the convex technology depicted as the shaded area in Figure 2. Note that DMU $B$ exhibits CRS and is at MPSS. DMU $A$ exhibits IRS and should increase 
the scale of its operations to achieve its MPSS at $B$. Therefore, $A$ exhibits G-IRS. Similarly, DMU $C$ exhibits DRS and G-DRS.

A related result by Banker et al. (1996) implies that the RTS and GRS characterizations are also equivalent in the VRS technology, which is nonsmooth. ${ }^{20}$ The recent development of various polyhedral technologies raises a question whether the equivalence of the corresponding RTS and GRS types could also be extended from the case of VRS to any such technology.

Below we prove that the answer to the above question is positive, and that this result is true not only for any polyhedral technology, but also for a larger class of closed convex technologies.

Let $\mathcal{T}$ be any closed convex technology, and let $\mathrm{DMU}_{o} \in \mathcal{T}$ satisfy Assumption 1.

Theorem 1. DMU exhibits G-CRS, G-IRS or G-DRS if and only if it exhibits local CRS, IRS or DRS, respectively. The G-SCRS type is therefore impossible.

Corollary 1. DMU $U_{o}$ is at MPSS if and only if it exhibits CRS.

The equivalence of MPSS and the CRS type of RTS stated by Corollary 1 is well known in the case of conventional VRS technology (Banker and Thrall, 1992). This means that, in the VRS technology, we can test for MPSS using two approaches. First, we can verify if the output radial efficiency of $\mathrm{DMU}_{o}$ in the reference CRS technology is equal to 1 , as in program (6). Second, we can verify if $\mathrm{DMU}_{o}$ exhibits CRS (and is therefore at MPSS) by calculating the one-sided scale elasticities $\varepsilon^{+}\left(X_{o}, Y_{o}\right)$ and $\varepsilon^{-}\left(X_{o}, Y_{o}\right)$ or, equivalently, the maximum and minimum values $\omega^{\max }$ and $\omega^{\min }$, as discussed in Section 4.

Corollary 1 implies that we have the same two choices in any closed convex technology (most importantly, in any polyhedral technology). In particular, instead of solving program (6), we can alternatively test for MPSS by evaluating the one-sided scale elasticities at $\mathrm{DMU}_{o}$. If $\mathcal{T}$ is a polyhedral technology, the latter approach is computationally straightforward and requires solving two linear programs (Podinovski et al., 2016). However, to solve program (6) we need to have an operational definition of the reference technology $\mathcal{T}^{*}$. Defining $\mathcal{T}^{*}$ may not be a straightforward task and may require additional research. Thus, such situation arises in the numerical example discussed in the next section. ${ }^{21}$

Furthermore, as noted in Section 6, the testing of types of GRS requires assessing the output radial efficiency of $\mathrm{DMU}_{o}$ in the reference NIRS and NDRS technologies generated by $\mathcal{T}$, and such technologies may not be immediately available either. However, as established by Theorem 1, we can equivalently test for local RTS types. As noted, if $\mathcal{T}$ is a polyhedral technology, this task is straightforward.

\section{Numerical example}

Theorem 1 establishes the equivalence of local and global RTS characterizations in any closed convex technology $\mathcal{T}$. In this section we illustrate this result by an example involving a VRS technology expanded by additional weight restrictions. DEA models based on such technologies are well established in the literature (see, e.g., Allen et al., 1997; Dyson and Thanassoulis, 1988; Podinovski, 2004d, 2005, 2016; Thanassoulis et al., 2014).

\footnotetext{
${ }^{20}$ See Podinovski (2004a, p. 245) for a discussion of this interpretation.

${ }^{21}$ Another example is the reference cone technology for the HRS technology (Podinovski, 2004c), which is developed in Podinovski (2009). Both technologies are subsequently used in the decomposition of the Malmquist index of productivity change in analysis of school efficiency reported in Podinovski et al. (2014).
} 


\subsection{Theoretical background}

Let observed DMUs be $\left(X_{j}, Y_{j}\right), j=1, \ldots, n$, where $X_{j} \in \mathbb{R}_{+}^{m}$ and $Y_{j} \in \mathbb{R}_{+}^{s}$ are the vectors of inputs and outputs respectively. To be specific, consider assessing the output radial efficiency of $\mathrm{DMU}_{o}$ by the multiplier VRS model. This model is stated in terms of variable vectors of input and output weights $v \in \mathbb{R}_{+}^{m}$ and $u \in \mathbb{R}_{+}^{s}$, respectively.

Weight restrictions are additional constraints on the input and output weights incorporated in the multiplier model. Suppose we have $K$ homogeneous weight restrictions stated in the following form:

$$
v^{\top} P_{t}-u^{\top} Q_{t} \geq 0, \quad t=1, \ldots, K .
$$

The output radial efficiency of $\mathrm{DMU}_{o}$ is the inverse of the optimal value $\eta^{*}$ of the following program:

$$
\begin{aligned}
\eta^{*}=\min & v^{\top} X_{o}+\omega \\
\text { subject to } & u^{\top} Y_{o}=1, \\
& v^{\top} X_{j}-u^{\top} Y_{j}+\omega \geq 0, \quad j=1, \ldots, n, \\
& v^{\top} P_{t}-u^{\top} Q_{t} \geq 0, \quad t=1, \ldots, K, \\
& u, v \geq 0, \omega \text { sign free. }
\end{aligned}
$$

The dual to program (8) is the output-oriented envelopment model which can be stated as follows:

$$
\begin{array}{ll}
\eta^{*}=\max & \eta \\
\text { subject to } & \sum_{j=1}^{n} \lambda_{j} X_{j}+\sum_{t=1}^{K} \pi_{t} P_{t} \leq X_{o}, \\
& \sum_{j=1}^{n} \lambda_{j} Y_{j}+\sum_{t=1}^{K} \pi_{t} Q_{t} \geq \eta Y_{o}, \\
& \sum_{j=1}^{n} \lambda_{j}=1, \\
& \lambda, \pi \geq 0, \eta \text { sign free, }
\end{array}
$$

where vector $\pi \in \mathbb{R}_{+}^{K}$ is dual to the weight restrictions in (8).

Observe that the envelopment model (9) includes the dual terms generated by weight restrictions in the multiplier model (8):

$$
\left(P_{t}, Q_{t}\right), \quad t=1, \ldots, K
$$

These terms, used in variable proportions $\pi_{t} \geq 0$, modify the DMUs in the standard VRS model represented by the first terms of constraints of program (9). Following Podinovski (2004d), the terms (10) are interpretable as production trade-offs between inputs and outputs. This implies that the envelopment program (9) assesses the output radial efficiency of $\mathrm{DMU}_{o}$ in the VRS technology expanded by production trade-offs (10). More precisely, this expanded technology is defined as follows:

Definition 4. (Podinovski, 2004d). The VRS technology with production trade-offs $\mathcal{T}_{\text {VRS-TO }}$ is the set of all nonnegative DMUs $(X, Y) \in \mathbb{R}_{+}^{m} \times \mathbb{R}_{+}^{s}$ for which there exist intensity 
Table 1: The data set in example.

\begin{tabular}{llllll}
\hline & Input 1 & Input 2 & Input 3 & Output 1 & Output 2 \\
\hline DMU 1 & 2 & 2 & 3 & 2 & 1 \\
DMU 2 & 4 & 4 & 3 & 4 & 3 \\
DMU 3 & 6 & 4 & 5 & 3 & 2 \\
DMU 4 & 2 & 3 & 3 & 3 & 4 \\
DMU 5 & 6 & 5 & 4 & 4 & 5 \\
\hline
\end{tabular}

vectors $\lambda \in \mathbb{R}_{+}^{n}, \pi \in \mathbb{R}_{+}^{K}$, and slack vectors $d \in \mathbb{R}_{+}^{m}$ and $e \in \mathbb{R}_{+}^{s}$ such that

$$
\begin{aligned}
& \sum_{j=1}^{n} \lambda_{j} X_{j}+\sum_{t=1}^{K} \pi_{t} P_{t}+d=X, \\
& \sum_{j=1}^{n} \lambda_{j} Y_{j}+\sum_{t=1}^{K} \pi_{t} Q_{t}-e=Y, \\
& \sum_{j=1}^{n} \lambda_{j}=1 .
\end{aligned}
$$

Technology $\mathcal{T}_{\text {VRS-TO }}$ is a polyhedral set and is, therefore, a convex technology (Podinovski, 2015).

Remark 5. It is well known that the incorporation of weight restrictions (7) in the multiplier model (8) may result in its infeasibility or, equivalently, in an unbounded solution to its dual envelopment model (9). Podinovski and Bouzdine-Chameeva (2013, 2015) refer to such weight restrictions as inconsistent (with the data set). They show that inconsistent weight restrictions generate either unlimited or free production of output vectors in technology $\mathcal{T}_{\text {VRS-TO }}{ }^{22}$ Moreover, it is possible that weight restrictions are inconsistent, even if for all observed DMUs, the corresponding multiplier model (8) is feasible, and the calculated efficiency scores appear unproblematic. Podinovski and Bouzdine-Chameeva $(2013,2015)$ develop simple necessary and sufficient conditions, and also computational approaches, for verifying the consistency of weight restrictions (7).

Below we assume that the weight restrictions (7) are consistent. Under this assumption, both the multiplier and envelopment models (8) and (9) have a finite optimal solution $\eta^{*}$, for any $\mathrm{DMU}_{o}$ in technology $\mathcal{T}_{\mathrm{VRS}-\mathrm{TO}}$, provided $Y_{o} \neq 0$.

\subsection{The data set and output radial efficiency}

The data set in Table 1 shows 5 DMUs assessed on 3 inputs and 2 outputs. Let $v_{1}, v_{2}$ and $v_{3}$ be the input weights, and $u_{1}$ and $u_{2}$ be the output weights. Consider assessing the output radial efficiency of the five DMUs using the VRS model with the following additional

\footnotetext{
${ }^{22}$ See footnotes 3 and 14 for the definitions of unlimited and free production.
} 
Table 2: One-sided scale elasticities $\varepsilon^{+}$and $\varepsilon^{-}$, and local RTS types of output radial efficient DMUs.

\begin{tabular}{llllll}
\hline & $\omega^{\min }$ & $\omega^{\max }$ & $\varepsilon^{-}$ & $\varepsilon^{+}$ & Type of RTS \\
\hline DMU 1 & $-\infty$ & -5 & $+\infty$ & 6 & IRS \\
DMU 2 & $-\infty$ & 0.3333 & $+\infty$ & 0.6667 & CRS \\
DMU 4 & $-\infty$ & 0.2118 & $+\infty$ & 0.7882 & CRS \\
DMU 5 & 0.1111 & 0.2727 & 0.8889 & 0.7273 & DRS \\
\hline
\end{tabular}

weight restrictions, which is a special case of $(7):^{23}$

$$
\begin{aligned}
& v_{1}-v_{2} \geq 0, \\
& -v_{1}+2 v_{2} \geq 0, \\
& -2 u_{1}+3 u_{2} \geq 0, \\
& v_{3}-2 u_{2} \geq 0 .
\end{aligned}
$$

The output radial efficiency of each DMU can be assessed by solving either the multiplier model (8) or its dual envelopment model (9). Computations show that four out of the five DMUs (all except DMU 3) are output radial efficient. All four such DMUs allow both the local and global RTS characterizations.

The output radial efficiency of DMU 3 is equal to 0.5. Therefore DMU 3 does not satisfy Assumption 1, and the notions of local and global RTS are undefined at this DMU.

\subsection{Local RTS}

As noted in Remark 2, the left-hand and right-hand scale elasticities $\varepsilon^{-}$and $\varepsilon^{+}$evaluated at any output radial efficient $\mathrm{DMU}_{o}$ can be calculated by formulae (4). ${ }^{24}$ For this we need to calculate the two extreme optimal values $\omega^{\min }$ and $\omega^{\max }$ of the sign free variable $\omega$ in the multiplier model. This task is straightforward and requires solving two linear programs for each $\mathrm{DMU}_{o}$, as shown in Appendix B.

Table 2 shows the values $\omega^{\text {min }}$ and $\omega^{\text {max }}$, and the corresponding one-sided scale elasticities evaluated at the four output radial efficient DMUs. By Definition 2, DMU 1 exhibits IRS, DMUs 2 and 4 exhibit CRS, and DMU 5 exhibits DRS. ${ }^{25}$

The obtained RTS characterization is by definition local, i.e., the RTS types are indicative of the proportion in which the output vector $Y_{o}$ of $\mathrm{DMU}_{o}$ would respond to marginal proportional changes of the input vector $X_{o}$, provided the resulting DMU remains output radial efficient. For example, DMU 5 exhibits DRS, and the right-hand scale elasticity evaluated at this DMU is equal to 0.7273 . This implies that, if we increase its input vector in a marginally small proportion, e.g., by $1 \%$, its output vector would increase more gradually, by $0.7273 \%$. If we reduce the input vector of this DMU by $1 \%$, the output vector would decrease by $0.8889 \%$.

\footnotetext{
${ }^{23}$ It is straightforward to verify that weight restrictions (12) are consistent. Indeed, the inequalities (12) are satisfied by the strictly positive weights $v_{1}=v_{2}=1, v_{3}=2$ and $u_{1}=u_{2}=1$. By Corollary 1 to Theorem 4 in Podinovski and Bouzdine-Chameeva (2015), the weight restrictions (12) are consistent.

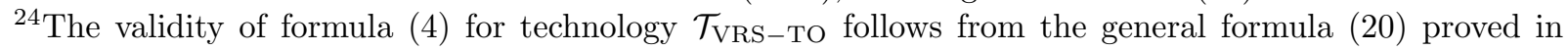
Podinovski et al. (2016).

${ }^{25}$ As established by Theorem 1 in Podinovski et al. (2016), the case $\varepsilon^{-}=+\infty$ (observed for DMUs 1,2 and 4) corresponds to the situation in which the input vector of $\mathrm{DMU}_{o}$ cannot be proportionally reduced, while keeping the resulting DMU in the technology.
} 


\subsection{Global RTS}

The question we are now concerned with is the following: are the types of local RTS discussed above (and shown in Table 2) indicative of the direction to MPSS for the four output-efficient DMUs, i.e., is the global characterization of RTS the same as the local one?

In the standard VRS model without additional weight restrictions, this question is positively answered by Proposition 1 in Banker (1984). For example, in the standard VRS technology (without weight restrictions), the IRS type indicates that the DMU should increase its scale of operations in order to reach its MPSS. If a DMU exhibits CRS, it is already at MPSS. On the other hand, this simple correspondence between the local and global characterizations generally breaks down if the technology is not convex (Podinovski, 2004a).

Theorem 1 gives a positive answer to the stated question. As noted, the VRS technology with weight restrictions is a convex technology. According to Theorem 1, the local RTS characterization of the output-efficient DMUs shown in Table 2 coincides with their global characterization. In particular, because DMU 1 exhibits local IRS, it also exhibits G-IRS and should increase the scale of its operations to achieve its MPSS. DMU 5 exhibits local DRS and therefore exhibits G-DRS, and should be scaled down to achieve its MPSS. DMUs 2 and 4 exhibit CRS and are therefore at their respective MPSS.

\subsection{Direct evaluation of GRS}

Taking into account Theorem 1, we could stop our investigation now because both the local and global characterizations of RTS have been obtained. However, for illustrative purposes, below we show how the global RTS types can be evaluated directly. This assessment (and the identification of DMUs at MPSS) requires additional computations utilizing the idea of the reference technology method (Färe et al. 1983, 1985). A variant of this method adjusted for testing for GRS types in an arbitrary technology was developed in Podinovski (2004a). This method relies on the evaluation of output radial efficiency of $\mathrm{DMU}_{o}$ in the NIRS and NDRS technologies generated by the underlying true technology, in our example technology $\mathcal{T}_{\text {VRS-TO. }}$

To the best of the author's knowledge, the DEA literature does not describe the required NIRS and NDRS technologies generated by technology $\mathcal{T}_{\text {VRS-TO. Following the general }}$ definition of Podinovski (2004a), let $\mathcal{C}, \mathcal{H}$ and $\mathcal{G}$ be the CRS (cone), NIRS and NDRS technologies generated by technology $\mathcal{T}_{\text {VRS-TO }}$. More precisely,

$$
\begin{aligned}
& \mathcal{C}=\left\{(X, Y) \in \mathbb{R}^{m+s} \mid \exists(\tilde{X}, \tilde{Y}) \in \mathcal{T}_{\text {VRS-TO }}, \delta \geq 0:(X, Y)=\delta(\tilde{X}, \tilde{Y})\right\}, \\
& \mathcal{H}=\left\{(X, Y) \in \mathbb{R}^{m+s} \mid \exists(\tilde{X}, \tilde{Y}) \in \mathcal{T}_{\text {VRS-TO }}, \delta \in[0,1]:(X, Y)=\delta(\tilde{X}, \tilde{Y})\right\}, \\
& \mathcal{G}=\left\{(X, Y) \in \mathbb{R}^{m+s} \mid \exists(\tilde{X}, \tilde{Y}) \in \mathcal{T}_{\text {VRS-TO }}, \delta \geq 1:(X, Y)=\delta(\tilde{X}, \tilde{Y})\right\} .
\end{aligned}
$$

In Appendix $\mathrm{C}$ we present examples that show that, generally, technologies $\mathcal{C}$ and $\mathcal{H}$ may not be closed sets. ${ }^{26}$ Let $\overline{\mathcal{C}}$ and $\overline{\mathcal{H}}$ be the closures of technologies $\mathcal{C}$ and $\mathcal{H}$, respectively. Denoting $\mathrm{cl}($.$) the closure operator, we have the following result.$

\footnotetext{
${ }^{26}$ If the VRS technology of Banker et al. (1984) is used without weight restrictions, its reference technologies $\mathcal{C}, \mathcal{H}$ and $\mathcal{G}$ are the standard CRS (Charnes et al., 1978), NIRS (Färe and Grosskopf, 1985) and NDRS (Seiford and Thrall, 1990) technologies. All these technologies are closed sets, and the particular problem with nonclosed reference technologies arising in the case of weight restrictions does not occur.
} 
Table 3: Output radial efficiency of DMUs in different technologies, all incorporating weight restrictions (12), and their GRS characterization. Note that DMU 3 is output radial inefficient, and its GRS type is undefined.

\begin{tabular}{llllll}
\hline & VRS & CRS & NIRS & NDRS & Type of GRS \\
\hline DMU 1 & 1 & 0.5 & 0.5 & 1 & G-IRS \\
DMU 2 & 1 & 1 & 1 & 1 & G-CRS (MPSS) \\
DMU 3 & 0.5 & 0.45 & 0.5 & 0.45 & Undefined \\
DMU 4 & 1 & 1 & 1 & 1 & G-CRS (MPSS) \\
DMU 5 & 1 & 0.9643 & 1 & 0.9643 & G-DRS \\
\hline
\end{tabular}

Theorem 2. (i) Technology $\overline{\mathcal{C}}=\operatorname{cl}(\mathcal{C})$ is described by conditions (11) from which the equality $(11 \mathrm{c})$ is removed. ${ }^{27}$

(ii) Technology $\overline{\mathcal{H}}=\operatorname{cl}(\mathcal{H})$ is described by conditions (11) in which equality (11c) is changed to the " $\leq$ " inequality.

(iii) Technology $\mathcal{G}$ is closed and described by conditions (11) in which equality (11c) is changed to the " $\geq$ " inequality.

Proposition 2. The output radial efficiency of any $D M U_{o} \in \mathcal{T}_{\mathrm{VRS}-\mathrm{TO}}$ evaluated in the reference technology $\mathcal{C}$ or $\mathcal{H}$ is equal to its output radial efficiency in the corresponding closed technology $\overline{\mathcal{C}}$ or $\overline{\mathcal{H}}$, respectively.

The above statement implies that in practice we can assess the efficiency of all DMUs in the closed technologies that have a simple operational form stated by Theorem $2 .{ }^{28}$

Table 3 shows the output radial efficiency of all five DMUs in the VRS technology $\mathcal{T}_{\text {VRS-TO }}$ and its reference CRS, NIRS and NDRS technologies. ${ }^{29}$ Appendix B shows an example of linear program used for the calculations.

Denote $E_{V R S}, E_{N I R S}$ and $E_{N D R S}$ the output radial efficiency of $\mathrm{DMU}_{o}$ in technologies $\mathcal{T}_{\text {VRS-TO }}, \mathcal{H}$ and $\mathcal{G}{ }^{30}$ By Theorem 3 in Podinovski (2004a), where we omit the fourth case of G-SCRS as impossible in a convex technology, DMU exhibits

(i) G-CRS if and only if $E_{N D R S}=E_{N I R S}=E_{V R S}$;

(iii) G-IRS if and only if $E_{N I R S}<E_{N D R S} \leq E_{V R S}$;

(ii) G-DRS if and only if $E_{N D R S}<E_{N I R S} \leq E_{V R S}$.

For example, as shown in Table 3, for DMU 1 we have: $E_{V R S}=1, E_{N I R S}=0.5$ and $E_{N D R S}=1$. Therefore, DMU 1 exhibits G-IRS. The last column of Table 3 shows the GRS types of all four output radial efficient DMUs. As formally established by Theorem 1, this is of course consistent with the local RTS characterization shown in Table 2.

\footnotetext{
${ }^{27}$ This means that $\overline{\mathcal{C}}=\operatorname{cl}(\mathcal{C})$ is the standard CRS technology of Charnes et al. (1978) expanded by the weight restrictions (7).

${ }^{28}$ Note that Proposition 2 is not a trivial statement. Thus, generally, the output radial efficiency of a $\mathrm{DMU}_{o}$ in an arbitrary technology $\mathcal{T}$ may be strictly smaller than in technology $\overline{\mathcal{T}}=\operatorname{cl}(\mathcal{T})$, even if $\overline{\mathcal{T}}$ is a polyhedral technology. An example of this is considered in Appendix C.

${ }^{29}$ The CRS technology is not required for the method of Podinovski (2004a) but is used by the method of Färe et al. $(1983,1985)$. We include technology $\mathcal{C}$ in Table 3 for completeness and reference purposes.

${ }^{30}$ Because DMUs $1,2,3$ and 4 are output radial efficient, we always have $E_{V R S}=1$ for each of them.
} 
In summary, as demonstrated, we can evaluate the local and global RTS types independently. However, as follows from Theorem 1, in a convex technology, the two characterizations are identical, and it suffices to obtain only one of them. An additional consideration here is that the local RTS characterization in any polyhedral technology can be obtained by the unifying approach developed in Podinovski et al. (2016), used in the above example. In contrast, as also shown by the above example, testing for global types of RTS requires operational statements of the NIRS and NDRS technologies, which may not be readily available.

\section{Further example: a two-stage network DEA model}

There is large literature on various types of network DEA models (see, e.g., Kao 2014). Consider the simple two-stage production process in which each DMU is described by the triplet $(X, Z, Y)$, where $X \in \mathbb{R}_{+}^{m}, Z \in \mathbb{R}_{+}^{p}$ and $Y \in \mathbb{R}_{+}^{s}$ are the vectors of inputs, intermediate outputs produced by the first production stage and acting as the inputs to the second production stage, and the vector of final outputs, respectively.

Each observed DMU $j=1, \ldots, n$ is stated as $\left(X_{j}, Z_{j}, Y_{j}\right)$. Denote $\bar{X}$ the $n \times m$ matrix whose columns are vectors $X_{j}, j=1, \ldots, n$. Similarly, let matrices $\bar{Z}$ and $\bar{Y}$ consist of the vector-columns $Z_{j}$ and $Y_{j}, j=1, \ldots, n$, respectively.

Suppose we are interested in the maximum production of the final outputs described by vector $Y$ from any given vector of inputs $X$. Taking into account the network structure of the production process, and assuming that both stages exhibit VRS, we define the technology $\mathcal{T}^{N}$ in the input and final output dimensions as follows. This definition is consistent with the definition used by Sahoo et al. (2014).

Definition 5. The network technology $\mathcal{T}^{N}$ with two VRS stages is the set of all nonnegative DMUs $(X, Y) \in \mathbb{R}_{+}^{m} \times \mathbb{R}_{+}^{s}$ for which there exist intensity vectors $\lambda, \mu \in \mathbb{R}_{+}^{n}$, intermediate outputs $Z \in \mathbb{R}_{+}^{p}$, and slack vectors $d \in \mathbb{R}_{+}^{m}, e \in \mathbb{R}_{+}^{s}, f, g \in \mathbb{R}_{+}^{p}$ such that

$$
\begin{aligned}
& \bar{X} \lambda+d=X, \\
& \bar{Y} \mu-e=Y, \\
& \bar{Z} \lambda-Z-f=0, \\
& \bar{Z} \mu-Z+g=0, \\
& 1^{\top} \lambda=1, \\
& 1^{\top} \mu=1 .
\end{aligned}
$$

The above conditions (14) are a special case of conditions (1) of Podinovski et al. (2016) defining a general polyhedral technology that satisfies free disposability of inputs and outputs. In particular, the latter general formulation utilizes vector $U_{o}$ important for the calculation of one-sided elasticities and RTS characterization. In our case vector $U_{o}$ is the vector of constants on the right-hand side of equalities (14c)-(14f), i.e.,

$$
U_{o}=(0,0,1,1)^{\top} .
$$

Note that the first two zero components of the vector $U_{o}$ are vectors, and the last two ones are scalars. ${ }^{31}$

\footnotetext{
${ }^{31}$ As required by formula (1) in Podinovski et al. (2016), we define the vector $\hat{\lambda}=(\lambda, \mu, Z, f, g)$. Then equalities (14a) and (14b) are special cases of conditions (1.1) and (1.2) of Podinovski et al. (2016). Equalities (14c)-(14f) are a special case of condition (1.3).
} 
Because $\mathcal{T}^{N}$ is a polyhedral technology, the calculation of the one-sided scale elasticities and many other marginal characteristics on its frontier can be performed by solving linear programs developed in Podinovski et al. (2016). In particular, let DMU $\left(X_{o}, Y_{o}\right)$ be output radial efficient in technology $\mathcal{T}^{N}$, i.e., satisfy the above Assumption 1.

Note that evaluating the output radial efficiency of $\mathrm{DMU}_{o}$ requires replacing vectors $X$ and $Y$ on the right-hand side of equations (14a) and (14b) by vectors $X_{o}$ and $\eta Y_{o}$, respectively, where $\eta$ is a sign-free scalar, and maximizing $\eta$ over the resulting constraints. ${ }^{32}$ Let $\mu, \nu$ and $\omega$ be the dual vectors corresponding to the constraints of this output-oriented linear program. More precisely, vectors $\mu$ and $\nu$ correspond to the constraints based on (14a) and (14b), and $\omega$ includes the dual variables to the constraints (14c)-(14f).

As shown by Podinovski et al. (2016), the one-sided scale elasticities at $\mathrm{DMU}_{o}$ in any polyhedral technology can be calculated as follows:

$$
\begin{aligned}
& \varepsilon^{+}\left(X_{o}, Y_{o}\right)=1-\max _{\langle\mu, \nu, \omega\rangle \in \Omega}\left\{U_{o}^{\top} \omega\right\}, \\
& \varepsilon^{-}\left(X_{o}, Y_{o}\right)=1-\min _{\langle\mu, \nu, \omega\rangle \in \Omega}\left\{U_{o}^{\top} \omega\right\},
\end{aligned}
$$

where $\Omega$ is the set of optimal solutions to the dual (multiplier) output-oriented program.

Taking into account (15), we can restate the general formula (16) by the following equivalent statement:

$$
\begin{aligned}
& \varepsilon^{+}\left(X_{o}, Y_{o}\right)=1-\max _{\langle\mu, \nu, \omega\rangle \in \Omega}\left\{\omega_{1}+\omega_{2}\right\}, \\
& \varepsilon^{-}\left(X_{o}, Y_{o}\right)=1-\min _{\langle\mu, \nu, \omega\rangle \in \Omega}\left\{\omega_{1}+\omega_{2}\right\},
\end{aligned}
$$

where $\omega_{1}$ and $\omega_{2}$ are the dual variables (scalars) to equalities (14e) and (14f), respectively.

According to Definition 2, the one-side scale elasticities (17) lead to the straightforward RTS characterization of $\mathrm{DMU}_{o}$. By Theorem 1, the local types of RTS stated by Definition 2 coincide with the corresponding GRS types. Furthermore, according to Corollary 1, a DMU in the network technology $\mathcal{T}^{N}$ is at MPSS if and only if it exhibits CRS.

It is worth emphasizing that obtaining these global characterizations of $\mathrm{DMU}_{o}$ would normally require constructing the NIRS and/or NDRS reference technologies for the network technology $\mathcal{T}^{N}$ which, to the best of the author's knowledge, are not readily available. However, as shown, the GRS types and related notion of MPSS can equivalently be evaluated using the notion of one-sided scale elasticities. Their evaluation in technology $\mathcal{T}^{N}$ requires solving two linear programs (17), which is a straightforward task.

Remark 6. The one-sided scale elasticities can also be obtained using the set of optimal solutions $\Delta$ to the dual (multiplier) input-oriented program. Assuming $\mathrm{DMU}_{o}$ is simultaneously input and output radial efficient, Podinovski et al. (2016, footnote 14) show that such scale elasticities are calculated as follows:

$$
\begin{aligned}
& \varepsilon^{+}\left(X_{o}, Y_{o}\right)=1 /\left(1-\min _{\langle\mu, \nu, \omega\rangle \in \Delta}\left\{U_{o}^{\top} \omega\right\}\right), \\
& \varepsilon^{-}\left(X_{o}, Y_{o}\right)=1 /\left(1-\max _{\langle\mu, \nu, \omega\rangle \in \Delta}\left\{U_{o}^{\top} \omega\right\}\right) .
\end{aligned}
$$

\footnotetext{
${ }^{32}$ In the case of input orientation, a similar program is considered in Sahoo et al. (2014).
} 
For the network technology $\mathcal{T}^{N}$, formulae (18) take on the form:

$$
\begin{aligned}
& \varepsilon^{+}\left(X_{o}, Y_{o}\right)=1 /\left(1-\min _{\langle\mu, \nu, \omega\rangle \in \Delta}\left\{\omega_{1}+\omega_{2}\right\}\right), \\
& \varepsilon^{-}\left(X_{o}, Y_{o}\right)=1 /\left(1-\max _{\langle\mu, \nu, \omega\rangle \in \Delta}\left\{\omega_{1}+\omega_{2}\right\}\right),
\end{aligned}
$$

where $\omega_{1}$ and $\omega_{2}$ are the dual variables to equalities (14e) and (14f), in the input-oriented multiplier program. This last expression generalizes formula (8) given in Banker and Thrall (1992) for the single-stage VRS technology.

\section{Conclusion}

In recent years, an important research avenue in DEA theory has been the development of new models based on more specific assumptions about the production process than those incorporated in the standard VRS model. Many of these new approaches model the production technology as a convex set and, almost invariably, as a polyhedral set. Examples of such polyhedral technologies arise in models with weakly disposable undesirable outputs, in extensions to the VRS technology by weight restrictions or production trade-offs, models of production processes with several components and network DEA models.

In a recent paper, Podinovski et al. (2016) developed a unifying linear programming methodology for the evaluation of one-sided scale elasticities and RTS characterization of DMUs in any polyhedral production technology. This development has led to a theoretical question, whether the local RTS characterization in an arbitrary polyhedral technology (conceptually defined by the scale elasticity evaluated at a given point on the frontier) is consistent with the global scale characteristics (including the notion of MPSS) as it is in the case of conventional VRS technology.

In this paper we address the above question in a more general class of closed convex technologies. We first establish that the one-sided scale elasticities of efficient frontiers in this class are "well-behaved", and their properties are sufficiently similar to those observed in polyhedral technologies. This allows us to extend the standard local RTS characterization to generally nonsmooth production frontiers of closed convex technologies. Based on this development, we prove that the types of RTS for this very large class of frontiers are consistent with the standard notions of MPSS and direction to MPSS expressed by global types of returns to scale.

The above development rigorously shows that closed convex technologies and, in particular, all polyhedral technologies are theoretically similar to the conventional VRS model. This result has an important practical implication. The standard methods of testing MPSS and, more generally, global types of RTS rely on the evaluation of output or input radial efficiency of $\mathrm{DMU}_{o}$ in the reference constant, nonincreasing and nondecreasing RTS technologies. In the conventional case of VRS, these technologies are well-known. However, for many other polyhedral technologies their reference technologies may not be readily available. Our theoretical results show that, instead of developing such reference technologies, we may alternatively evaluate the types of RTS using the existing linear programming methodologies suitable for any polyhedral technology. Based on results of this paper, the task of translating the types of local RTS into the MPSS and global RTS types becomes straightforward. 


\section{Appendix A. Proofs}

Proof of Proposition 1. The proof of part (i) follows from the assumption that $\mathcal{T}$ is a closed convex set. To prove part (ii), first note that $\sup \beta(\alpha)<+\infty$, for all $\alpha \in \Gamma$. Indeed, assume the opposite, i.e., that there exists an $\alpha^{*} \in \Gamma$ such that $\sup \beta\left(\alpha^{*}\right)=+\infty$. Then by Theorem 2 in Podinovski and Bouzdine-Chameeva (2013), $\sup \beta(\alpha)=+\infty$, for all $\alpha \in \Gamma$, which contradicts Assumption 1. Therefore, for every $\alpha \in \Gamma, \sup \beta(\alpha)$ is finite and, because $\mathcal{T}$ is closed, it is attained. Part (iii) is proved as Lemma 3 in Podinovski (2004a).

We now prove Corollary 1 as a lemma, before proving Theorem 1.

Lemma 1. DMU $U_{o}$ is at MPSS if and only if it exhibits CRS.

Proof of Lemma 1. Let $\mathrm{DMU}_{o}$ exhibit CRS. We need to prove that $\mathrm{DMU}_{o}$ is at MPSS, i.e.,

$$
\frac{\bar{\beta}(\alpha)}{\alpha} \leq 1, \forall \alpha \in \Gamma, \alpha>0
$$

Let $\alpha=1$ be an interior point of $\Gamma$. Then, as noted in Section 2, the one-sided derivatives $\bar{\beta}_{-}^{\prime}(1)$ and $\bar{\beta}_{+}^{\prime}(1)$ exist and are finite. For all $\alpha \in \Gamma$ we have

$$
\begin{aligned}
& \bar{\beta}(\alpha) \leq 1+\bar{\beta}_{+}^{\prime}(1)(\alpha-1), \\
& \bar{\beta}(\alpha) \leq 1+\bar{\beta}_{-}^{\prime}(1)(\alpha-1) .
\end{aligned}
$$

Consider any $\alpha \in \Gamma$. If $\alpha>1$ then, because $\bar{\beta}_{+}^{\prime}(1) \leq 1$, (A.2a) implies $\bar{\beta}(\alpha) \leq \alpha$. If $0<\alpha<1$ then, because $\bar{\beta}_{-}^{\prime}(1) \geq 1,($ A.2b) implies $\bar{\beta}(\alpha) \leq \alpha$. Both cases imply (A.1).

Now assume that $\alpha=1$ is the left extreme point of $\Gamma$. Then $\alpha \geq 1$ for all $\alpha \in \Gamma$. As noted in Section $2, \bar{\beta}_{+}^{\prime}(1)>-\infty$. Taking into account the definition of CRS, $\bar{\beta}_{+}^{\prime}(1)$ is finite and $\bar{\beta}_{+}^{\prime}(1) \leq 1$. The inequality (A.1) now follows from (A.2a) as above.

Let $\alpha=1$ be the right extreme point of $\Gamma$. We need to prove (A.1) for all $\alpha \in \Gamma$, $0<\alpha<1$. As noted in Section $2, \bar{\beta}_{-}^{\prime}(1)<+\infty$. Taking into account the definition of CRS, $\bar{\beta}_{-}^{\prime}(1)$ is finite and $\bar{\beta}_{-}^{\prime}(1) \geq 1$. Then (A.1) follows from (A.2b), as above.

Conversely, assume that $\mathrm{DMU}_{o}$ is at MPSS, i.e., (A.1) is true. We need to prove that $\bar{\beta}_{+}^{\prime}(1) \leq 1$, if $\alpha=1$ is not the right extreme point of $\Gamma$, and $\bar{\beta}_{-}^{\prime}(1) \geq 1$, if $\alpha=1$ is not the left extreme point of $\Gamma$. Both proofs are similar, and we prove only the first part. By definition, we have

$$
\bar{\beta}_{+}^{\prime}(1)=\lim _{\alpha \downarrow 1} \frac{\bar{\beta}(\alpha)-\bar{\beta}(1)}{\alpha-1} .
$$

Assume that $\bar{\beta}_{+}^{\prime}(1)>1$. Then for all $\alpha>1$ sufficiently close to 1 we have

$$
\frac{\bar{\beta}(\alpha)-\bar{\beta}(1)}{\alpha-1}>1
$$

By rearrangement and because $\bar{\beta}(1)=1$, we have $\bar{\beta}(\alpha)>\alpha$, which contradicts (A.1).

Proof of Theorem 1. The equivalence of G-CRS and CRS types is established by Lemma 1. For further proof, consider the function of ray average productivity $\varphi(\alpha)=\bar{\beta}(\alpha) / \alpha$ defined on $\Gamma_{0}=\Gamma \backslash\{0\}$. Denote $\varphi^{*}$ the supremum of $\varphi(\alpha)$ on $\Gamma_{0}$. Note that $\varphi^{*}$ may be finite or equal to $+\infty$, and may be attained or unattained. Denote $\Gamma^{*}=\left\{\alpha \in \Gamma \mid \varphi(\alpha)=\varphi^{*}\right\}$. $\Gamma^{*}=\varnothing$ if $\varphi^{*}$ is not attained. By Lemma 5 in Podinovski (2004a), if $\Gamma^{*} \neq \varnothing$ then $\Gamma^{*}$ is a closed interval. Therefore, we can represent $\Gamma_{0}$ as the union of three intervals as follows:

$$
\Gamma_{0}=\Gamma^{+} \cup \Gamma^{*} \cup \Gamma^{-},
$$


where, for any $\alpha_{1} \in \Gamma^{+}, \alpha_{2} \in \Gamma^{*}$ and $\alpha_{3} \in \Gamma^{-}$, we have $\alpha_{1}<\alpha_{2}<\alpha_{3}$. Any of the three intervals $\Gamma^{+}, \Gamma^{*}$ and $\Gamma^{-}$, but not all of them, may be empty sets, however, the case $\Gamma^{*}=\varnothing$, while $\Gamma^{+} \neq \varnothing$ and $\Gamma^{-} \neq \varnothing$, is impossible (Podinovski 2004a).

By Theorem 5 proved in Podinovski (2004a), $\varphi(\alpha)$ is strictly increasing on $\Gamma^{+}$and strictly decreasing on $\Gamma^{-}$(provided these intervals are not empty). This implies that $\mathrm{DMU}_{o}$ exhibits G-IRS if $1 \in \Gamma^{+}$, G-CRS (and hence is at MPSS) if $1 \in \Gamma^{*}$, and G-DRS if $\alpha=1 \in \Gamma^{-}$.

Let $1 \in \Gamma^{+}$. Because $\Gamma^{*}$ is a closed interval, from (A.5), $\alpha=1$ is not the right extreme point of $\Gamma^{+}$. Because $\varphi(\alpha)$ is strictly increasing on $\Gamma^{+}$, for all $\alpha$ located in $\Gamma^{+}$to the right of $\alpha=1$, we have $\bar{\beta}(\alpha) / \alpha>\bar{\beta}(1) / 1=1$. This implies (A.4). By (A.3), $\bar{\beta}_{+}^{\prime}(1) \geq 1$. If we assume that $\bar{\beta}_{+}^{\prime}(1)=1$, then by Definition $2, \mathrm{DMU}_{o}$ exhibits CRS and, by Lemma $1, \mathrm{DMU}_{o}$ is at MPSS. Therefore $1 \in \Gamma^{*}$, which contradicts the assumption. Therefore $\bar{\beta}_{+}^{\prime}>1$ and, by Definition 2, $\mathrm{DMU}_{o}$ exhibits IRS.

If $1 \in \Gamma^{-}$, a similar proof establishes that $\mathrm{DMU}_{o}$ exhibits DRS.

Proof of Theorem 2. Consider statement (i). Denote $\mathcal{C}^{*}$ the set of DMUs $(X, Y)$ described by conditions (11) from which the equality (11c) is removed. We need to prove that $\overline{\mathcal{C}}=\mathcal{C}^{*}$. We first note that $\mathcal{C}^{*}$ is a polyhedral set (Theorem 3 in Podinovski, 2015). Therefore, $\mathcal{C}^{*}$ is a closed set.

Consider any $\operatorname{DMU}(X, Y) \in C$. By $(13),(X, Y)=\delta(\tilde{X}, \tilde{Y})$, where $(\tilde{X}, \tilde{Y}) \in \mathcal{T}_{\text {VRS-To }}$. The latter DMU satisfies (11) with some vectors $\tilde{\lambda}, \tilde{\pi}$, $\tilde{e}$ and $\tilde{d}$. Then $\operatorname{DMU} \delta(\tilde{X}, \tilde{Y})$ satisfies (11a) and (11b) with the vectors $\delta \tilde{\lambda}, \delta \tilde{\pi}, \delta \tilde{e}$ and $\delta \tilde{d}$. Therefore, $(X, Y) \in \mathcal{C}^{*}$, and $\mathcal{C} \subseteq \mathcal{C}^{*}$. Because the set $\mathcal{C}^{*}$ is closed, we have $\overline{\mathcal{C}}=\operatorname{cl}(\mathcal{C}) \subseteq \operatorname{cl}\left(\mathcal{C}^{*}\right)=\mathcal{C}^{*}$.

Conversely, let $(X, Y) \in \mathcal{C}^{*}$. Then $(X, Y)$ satisfies (11a) and (11b) with some vectors $\lambda^{\prime}$, $\pi^{\prime}, e^{\prime}$ and $d^{\prime}$. Denote $\lambda^{*}=1^{\top} \lambda^{\prime}$. The following two cases are possible. First, let $\lambda^{*}>0$. Define $(\tilde{X}, \tilde{Y})=\left(1 / \lambda^{*}\right)(X, Y)$. Then $(\tilde{X}, \tilde{Y})$ satisfies (11) with $\lambda=\lambda^{\prime} / \lambda^{*}, \pi=\pi^{\prime} / \lambda^{*}$, $e=e^{\prime} / \lambda^{*}$ and $d=d^{\prime} / \lambda^{*}$. Therefore, $(\tilde{X}, \tilde{Y}) \in \mathcal{T}_{\text {VRS-TO. By }}(13),(X, Y) \in \mathcal{C} \subseteq \overline{\mathcal{C}}$.

Now let $\lambda^{*}=0$. Therefore, $\lambda^{\prime}=0$. Consider DMUs $\left(X_{k}, Y_{k}\right), k=1,2, \ldots$, defined as follows:

$$
\left(X_{k}, Y_{k}\right)=\sum_{j=1}^{n}\left(\frac{1}{n}\left(X_{j}, Y_{j}\right)\right)+k(X, Y) .
$$

Each DMU $\left(X_{k}, Y_{k}\right)$ is nonnegative and satisfies all conditions (11) with the vectors $\lambda_{k}=(1 / n, \ldots, 1 / n), \pi_{k}=k \pi^{\prime}, e_{k}=k e^{\prime}$ and $d_{k}=k d^{\prime}$. Therefore, $\left(X_{k}, Y_{k}\right) \in \mathcal{T}_{\text {VRS-TO }}$, for all $k=1,2, \ldots$ Consider the sequence of DMUs $\left(\tilde{X}_{k}, \tilde{Y}_{k}\right)=(1 / k)\left(X_{k}, Y_{k}\right)$. By (13), we have $\left(\tilde{X}_{k}, \tilde{Y}_{k}\right) \in \mathcal{C}$, for all $k=1,2, \ldots$ Furthermore,

$$
\lim _{k \rightarrow+\infty}\left(\tilde{X}_{k}, \tilde{Y}_{k}\right)=(X, Y)
$$

Therefore, $(X, Y) \in \overline{\mathcal{C}}$. Because $\operatorname{DMU}(X, Y)$ is an arbitrary element of $\mathcal{C}^{*}$, in both cases $\lambda^{*}>0$ and $\lambda^{*}=0$ we have $\overline{\mathcal{C}} \subseteq \overline{\mathcal{C}}$. Taking into account the first part of the proof, we have $\mathcal{C}^{*}=\overline{\mathcal{C}}$.

The proof of the other two statements of the theorem is similar. It requires defining technologies $\mathcal{H}^{*}$ and $\mathcal{G}^{*}$ as the sets of all DMUs that satisfy conditions (11) in which the equality (11c) is replaced by the " $\leq$ " and " $\geq$ " inequality, respectively. As in the case of technology $\mathcal{C}^{*}$, it is first established that the sets $\mathcal{H}^{*}$ and $\mathcal{G}^{*}$ are polyhedral and therefore closed. Repeating the steps of the above proof, it is straightforward to establish that $\mathcal{H}^{*}=\overline{\mathcal{H}}$. Because for technology $\mathcal{G}$ the case $\lambda^{\prime}=0$ is impossible, we have $\mathcal{G}^{*}=\mathcal{G}$, which implies that $\mathcal{G}$ is a closed set. 
Proof of Proposition 2. Define $\eta^{*}=\sup \left\{\eta \mid\left(X_{o}, \eta Y_{o}\right) \in \mathcal{C}\right\}$ and $\bar{\eta}^{*}=\sup \left\{\eta \mid\left(X_{o}, \eta Y_{o}\right) \in\right.$ $\overline{\mathcal{C}}\}$. Because $\mathcal{C} \subseteq \overline{\mathcal{C}}$, we have $\eta^{*} \leq \bar{\eta}^{*}$.

Conversely, note that the weight restrictions (7) are assumed to be consistent in the VRS technology $\mathcal{T}_{\text {VRS-TO }}$. By Theorem 5 in Podinovski and Bouzdine-Chameeva (2013), they are also consistent in technology $\overline{\mathcal{C}}$ which, as noted in footnote 27 , is the CRS technology with weight restrictions (7). Therefore, the supremum $\bar{\eta}^{*}<+\infty$. Because technology $\overline{\mathcal{C}}$ is closed, $\bar{\eta}^{*}$ is attained. We also obviously have $\bar{\eta}^{*} \geq 1$ and $\left(X_{o}, \bar{\eta}^{*} Y_{o}\right) \in \overline{\mathcal{C}}$.

Let DMU $\left(X_{o}, Y_{o}\right) \in \mathcal{T}_{\mathrm{VRS}-\mathrm{TO}}$ satisfy $(11)$ with some vectors $\tilde{\lambda}, \tilde{\pi}$, $\tilde{e}$ and $\tilde{d}$. Similarly, let $\operatorname{DMU}\left(X_{o}, \bar{\eta}^{*} Y_{o}\right) \in \overline{\mathcal{C}}$ satisfy conditions (11a) and (11b) with some vectors $\lambda^{\prime}, \pi^{\prime}, e^{\prime}$ and $d^{\prime}$. Let $\lambda^{*}=1^{\top} \lambda^{\prime}$. Two cases arise.

First, let $\lambda^{*}>0$. In this case, it is straightforward to prove that $\left(X_{o}, \bar{\eta}^{*} Y_{o}\right) \in \mathcal{C}$. Indeed, define $(\tilde{X}, \tilde{Y})=\left(1 / \lambda^{*}\right)\left(X_{o}, \bar{\eta}^{*} Y_{o}\right)$. Then $(\tilde{X}, \tilde{Y})$ satisfies (11) with $\lambda=\lambda^{\prime} / \lambda^{*}, \pi=\pi^{\prime} / \lambda^{*}$, $e=e^{\prime} / \lambda^{*}$ and $d=d^{\prime} / \lambda^{*}$. Therefore, $(\tilde{X}, \tilde{Y}) \in \mathcal{T}_{\text {VRS-To. Because }}\left(X_{o}, \bar{\eta}^{*} Y_{o}\right)=\lambda^{*}(\tilde{X}, \tilde{Y})$, by $(13),\left(X_{o}, \bar{\eta}^{*} Y_{o}\right) \in \mathcal{C}$. Therefore, if $\lambda^{*}>0$, we have $\eta^{*} \geq \bar{\eta}^{*}$.

Now let $\lambda^{*}=0$. For all $k=1,2, \ldots$ define

$$
\left(\tilde{X}_{k}, \tilde{Y}_{k}\right)=\frac{1}{k}\left(X_{o}, Y_{o}\right)+\left(1-\frac{1}{k}\right)\left(X_{o}, \bar{\eta}^{*} Y_{o}\right)
$$

Each DMU $\left(\tilde{X}_{k}, \tilde{Y}_{k}\right)$ is nonnegative and satisfies (11a) and (11b) with the vectors $\lambda_{k}=$ $(1 / k) \tilde{\lambda}, \pi_{k}=(1 / k) \tilde{\pi}+(1-1 / k) \pi^{\prime}, e_{k}=(1 / k) \tilde{e}+(1-1 / k) e^{\prime}$ and $d_{k}=(1 / k) \tilde{d}+(1-1 / k) d^{\prime}$. Therefore, $\left(\tilde{X}_{k}, \tilde{Y}_{k}\right) \in \mathcal{C}$, for all $k=1,2, \ldots$ Rearranging (A.6), we have

$$
\left(\tilde{X}_{k}, \tilde{Y}_{k}\right)=\left(X_{o}, \eta_{k} Y_{o}\right) \in \mathcal{C}
$$

where $\eta_{k}=1 / k+(1-1 / k) \bar{\eta}^{*} \leq \bar{\eta}^{*}$. Because $\lim _{k \rightarrow+\infty} \eta_{k}=\bar{\eta}^{*}$, and by definition of $\eta^{*}$, we have $\eta^{*} \geq \bar{\eta}^{*}$. Combining all parts of the above proof, we have $\eta^{*}=\bar{\eta}^{*}$.

The proof for technologies $\mathcal{H}$ and $\overline{\mathcal{H}}$ is similar. In particular, because $\overline{\mathcal{H}} \subseteq \overline{\mathcal{C}}$, we have $\hat{\eta}^{*}=\sup \left\{\eta \mid\left(X_{o}, \eta Y_{o}\right) \in \overline{\mathcal{H}}\right\} \leq \bar{\eta}^{*}$. As proved, $\bar{\eta}^{*}<+\infty$. Therefore, $\hat{\eta}^{*}<+\infty$. The rest of the proof is similar to the proof for technology $\overline{\mathcal{C}}$ and is omitted. 


\section{Appendix B. Models for the numerical example}

Below we present linear programs used in the assessment of local and global RTS in the example discussed in Section 8. To be specific, consider DMU 1.

To calculate the one-sided scale elasticities at DMU 1, we first evaluate the extreme values of variable $\omega$ in the set of optimal solutions to the corresponding program (8). For example, the value $\omega^{\max }$ is obtained by solving the following linear program: ${ }^{33}$

$$
\begin{aligned}
\omega^{\max }=\max & \omega \\
\text { subject to } & 2 v_{1}+2 v_{2}+3 v_{3}+\omega=1, \\
& 2 u_{1}+1 u_{2}=1, \\
& 2 v_{1}+2 v_{2}+3 v_{3}-2 u_{1}-1 u_{2}+\omega \geq 0, \\
& 4 v_{1}+4 v_{2}+3 v_{3}-4 u_{1}-3 u_{2}+\omega \geq 0, \\
& 6 v_{1}+4 v_{2}+5 v_{3}-3 u_{1}-2 u_{2}+\omega \geq 0, \\
& 2 v_{1}+3 v_{2}+3 v_{3}-3 u_{1}-4 u_{2}+\omega \geq 0, \\
& 6 v_{1}+5 v_{2}+4 v_{3}-4 u_{1}-5 u_{2}+\omega \geq 0, \\
& v_{1}-v_{2} \geq 0, \\
& -v_{1}+2 v_{2} \geq 0, \\
& -2 u_{1}+3 u_{2} \geq 0, \\
& v_{3}-2 u_{2} \geq 0, \\
& u_{1}, u_{2}, v_{1}, v_{2}, v_{3} \geq 0, \omega \text { sign free. }
\end{aligned}
$$

The value $\omega^{\text {min }}$ is obtained by changing the maximization of $\omega$ in the above program to its minimization. We convert the obtained values $\omega^{\max }$ and $\omega^{\min }$ to the one-sided scale elasticities using formula (4).

For the GRS characterization, we first assess the output radial efficiency of each $\mathrm{DMU}_{o}$ in the reference NIRS and NDRS technologies for technology $\mathcal{T}_{\text {VRS-TO. As described in }}$ Section 8, the GRS type of each $\mathrm{DMU}_{o}$ is obtained by comparing these efficiencies with its efficiency in technology $\mathcal{T}_{\text {VRS-TO }}$.

For example, the output radial efficiency of DMU 1 in the reference NIRS technology $\mathcal{H}$ (more precisely, in its closure $\overline{\mathcal{H}}$ : see Section 8 for a discussion) is the inverse to the optimal value $\eta^{*}$ of the following program:

$$
\begin{aligned}
& \eta^{*}=\max \quad \eta \\
& \text { subject to } \\
& 2 \lambda_{1}+4 \lambda_{2}+6 \lambda_{3}+2 \lambda_{4}+6 \lambda_{5}+1 \pi_{1}-1 \pi_{2} \leq 2, \\
& 2 \lambda_{1}+4 \lambda_{2}+4 \lambda_{3}+3 \lambda_{4}+5 \lambda_{5}-1 \pi_{1}+2 \pi_{2} \leq 2 \text {, } \\
& 3 \lambda_{1}+3 \lambda_{2}+5 \lambda_{3}+3 \lambda_{4}+4 \lambda_{5}+1 \pi_{4} \leq 3 \text {, } \\
& 2 \lambda_{1}+4 \lambda_{2}+3 \lambda_{3}+3 \lambda_{4}+4 \lambda_{5}+2 \pi_{3} \geq 2 \eta \text {, } \\
& 1 \lambda_{1}+3 \lambda_{2}+2 \lambda_{3}+4 \lambda_{4}+5 \lambda_{5}-3 \pi_{3}+2 \pi_{4} \geq 1 \eta \text {, } \\
& 1 \lambda_{1}+1 \lambda_{2}+1 \lambda_{3}+1 \lambda_{4}+1 \lambda_{5} \leq 1, \\
& \lambda_{1}, \lambda_{2}, \lambda_{3}, \lambda_{4}, \lambda_{5}, \pi_{1}, \pi_{2}, \pi_{3}, \pi_{4} \geq 0 \text {. }
\end{aligned}
$$

For the output radial efficiency in the reference NDRS technology $\mathcal{G}$, we change the sign of the second last inequality in the above program to " $\geq$ ".

\footnotetext{
${ }^{33}$ This program maximises $\omega$ subject to the same constraints as in program (8) and the additional first constraint that keeps the objective function of program (8) equal to 1.
} 


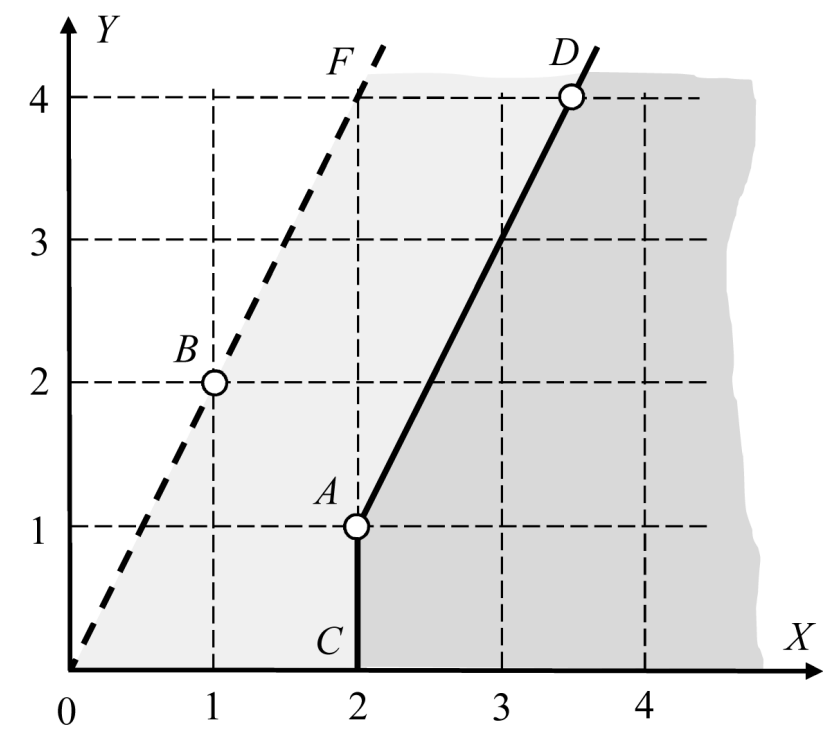

Figure C.3: Technology $\mathcal{T}_{\text {VRS-TO }}$ (shown in dark grey) for which its reference CRS and NIRS technologies (expanded by the light grey area) are the same and are not closed sets.

\section{Appendix C. Examples}

Below we give an example of a VRS technology with weight restrictions $\mathcal{T}_{\text {VRS-TO }}$ whose CRS (cone) and NIRS reference technologies $\mathcal{C}$ and $\mathcal{H}$ are not closed sets. We also give an example that illustrates footnote 28. These examples underline the importance of Theorem 2 and Proposition 2 for the assessment of output radial efficiency in these reference technologies.

Figure C.3 shows the single observed DMU $A$ whose input $X$ is equal to 2, and output $Y$ is equal to 1 . Assume that we have specified the weight restriction

$$
v_{1}-2 u_{1} \geq 0 \text {. }
$$

By Definition 4 , the VRS technology $\mathcal{T}_{\text {VRS-TO }}$ induced by DMU $A$ and weight restriction (C.1) is the set of nonnegative DMUs $(X, Y)$ such that, restating (11), we trivially have:

$$
\begin{aligned}
& 2 \lambda_{1}+1 \pi_{1}+d_{1}=X, \\
& 1 \lambda_{1}+2 \pi_{1}-e_{1}=Y, \\
& 1 \lambda_{1}=1, \\
& \lambda_{1}, \pi_{1}, d_{1}, e_{1} \geq 0 .
\end{aligned}
$$

Technology $\mathcal{T}_{\text {VRS-TO }}$ is shown in Figure C.3 as the dark grey area below the ray $A D$. For example, DMU $D$ is obtained from (C.2) by taking $\lambda_{1}=1, \pi_{1}=1.5$, and $d_{1}=e_{1}=0$.

The reference CRS technology. According to (13), the reference CRS (cone) technology $\mathcal{C}$ is obtained from technology $\mathcal{T}_{\text {VRS-TO }}$ by scaling its units by arbitrary multipliers $\delta \geq 0$. Therefore, technology $\mathcal{C}$ includes all rays starting from the origin $O$ and passing through an arbitrary point in $\mathcal{T}_{\text {VRS-TO }}$. For example, $\mathcal{C}$ includes the rays $O A$ and $O D$. From Figure C.3 it is clear that technology $\mathcal{C}$ is the set under the ray $O F$ (shown in both light and dark grey shading). Note that technology $\mathcal{C}$ does not include the ray $O F$ and is not a closed set. 
The closure of technology $\mathcal{C}$, denoted $\overline{\mathcal{C}}$, is the closed cone that includes the ray $O F$ and the shaded area below it. This technology is described by conditions (C.2) from which the equality $1 \lambda_{1}=1$ is removed. For example, DMU $B$ satisfies the resulting conditions with $\lambda_{1}=0, \pi_{1}=1$, and $d_{1}=e_{1}=0$. This illustrates statement (i) of Theorem 2 .

Note that technology $\overline{\mathcal{C}}$ is (but technology $\mathcal{C}$ is not) the conventional CRS technology expanded by the incorporation of weight restrictions (C.1). ${ }^{34}$

The reference NIRS technology. According to definition (13), the reference NIRS technology $\mathcal{H}$ is obtained by scaling DMUs in technology $\mathcal{T}_{\text {VRS-TO }}$ by multipliers $\delta \in[0,1]$. It is clear that, for the technology in Figure C.3, its reference NIRS technology $\mathcal{H}$ coincides with the reference $\mathrm{CRS}$ technology $\mathcal{C}$ discussed above. Therefore, technology $\mathcal{H}$ is the shaded area below the ray $O F$, which excludes this ray and is not therefore a closed set. Its closure is technology $\overline{\mathcal{H}}$ which includes the ray $O F$ and the shaded area below it.

As proved by statement (ii) of Theorem 2, the closed technology $\overline{\mathcal{H}}$ is described by conditions (C.2) in which the normalizing equality for $\lambda_{1}$ is replaced by the inequality $1 \lambda_{1} \leq 1$. As in the case of CRS technology, DMU $B$ and any other DMU on or below the ray $O F$ satisfies the resulting conditions with appropriately selected variables $\lambda_{1}, \pi_{1}, d_{1}$ and $e_{1}$.

Technology $\overline{\mathcal{H}}$ (but not the technology $\mathcal{H}$ ) can be regarded as the conventional NIRS technology (Färe and Grosskopf, 1985) expanded by weight restrictions (C.1).

The reference NDRS technology. Using definition (13), the reference NDRS technology $\mathcal{G}$ is obtained by scaling all DMUS in technology $\mathcal{T}_{\text {VRS-TO }}$ by multipliers $\delta \geq 1$. In our case,

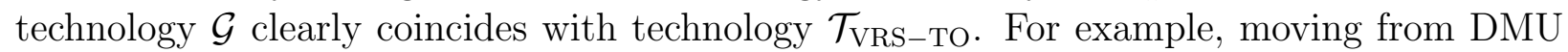
$A$ along the ray $O A$ and away from the origin (which corresponds to scaling DMU $A$ with $\delta \geq 1$ ) generates DMUs that are already in technology $\mathcal{T}_{\text {VRS-TO }}$

According to statement (iii) of Theorem 2, technology $\mathcal{G}$ is described by conditions (C.2) in which the normalizing equality for $\lambda_{1}$ is replaced by the inequality $1 \lambda_{1} \geq 1$. It is clear that, in this particular example, the resulting relaxed set of conditions does not generate any new hypothetical (unobserved) DMUs compared to technology $\mathcal{T}_{\text {VRS-TO }}$.

As proved by Theorem 2 and illustrated by this example, technology $\mathcal{G}$ is a closed set. The reference NDRS technology $\mathcal{G}$ can be regarded as the conventional NDRS technology (Seiford and Thrall, 1990) expanded by weight restrictions (C.1).

\section{Example illustrating Proposition 2.}

Consider technology $\mathcal{T}$ shown as the shaded area in Figure C.4. Note that $\mathcal{T}$ includes the segment $A C$ but excludes all points strictly between $A$ and $B$, and also excludes $B$. The closure $\overline{\mathcal{T}}=\operatorname{cl}(\mathcal{T})$ includes the entire segment $B C$.

The output radial efficiency of $\operatorname{DMU} A=(1,1)$ in technology $\mathcal{T}$ is the inverse of the supremum

$$
\eta^{*}=\sup \{\eta \mid(1, \eta) \in \mathcal{T}\}=1
$$

Therefore, the output radial efficiency of DMU $A$ in $\mathcal{T}$ is equal to 1 . This is strictly smaller than the output radial efficiency of $A$ in technology $\overline{\mathcal{T}}$, which is equal to 0.5 .

\footnotetext{
${ }^{34}$ A standard definition of the CRS technology with weight restrictions is given by Podinovski (2004d). It is obtained from Definition 4 by removing condition (11c).
} 


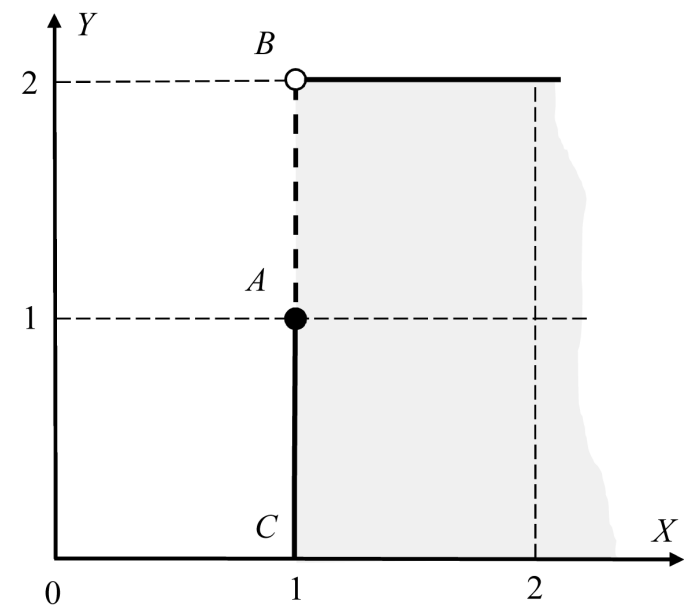

Figure C.4: Example of technology $\mathcal{T}$ in which the output radial efficiency of DMU $A$ is strictly smaller than in technology $\overline{\mathcal{T}}=\operatorname{cl}(\mathcal{T})$.

\section{References}

Allen, R., Athanassopoulos, A., Dyson, R.G., Thanassoulis, E. (1997). Weights restrictions and value judgements in data envelopment analysis: Evolution, development and future directions. Annals of Operations Research, 73, 13-34.

Atici, K.B., Podinovski, V.V. (2012). Mixed partial elasticities in constant returns-to-scale production technologies. European Journal of Operational Research, 220(1), 262-269.

Atici, K.B., Podinovski, V.V. (2015). Using data envelopment analysis for the assessment of technical efficiency of units with different specialisations: An application to agriculture. Omega, 54, 72-83.

Banker, R.D. (1984). Estimating most productive scale size using data envelopment analysis. European Journal of Operational Research, 17(1), 35-44.

Banker, R.D., H. Chang, H., Cooper, W.W. (1996). Equivalence and implementation of alternative methods for determining returns to scale in data envelopment analysis. European Journal of Operational Research, $89(3), 473-481$.

Banker, R.D., Charnes, A., Cooper, W.W. (1984). Some models for estimating technical and scale efficiencies in data envelopment analysis. Management Science, 30(9), 1078-1092.

Banker, R.D., Thrall, R.M. (1992). Estimation of returns to scale using data envelopment analysis. European Journal of Operational Research, 62(1), 74-84.

Banker, R.D., Cooper, W.W., Seiford, L.M., Zhu, J. (2011). Returns to scale in DEA. In W.W. Cooper, L.M, Seiford, J. Zhu (Eds.), Handbook on data envelopment analysis. (2nd ed.). (pp. 41-70). New York: Springer Science + Busines Media.

Briec, W., Kerstens, K., Leleu, H., Vanden Eeckaut, P. (2000) Returns to scale on nonparametric deterministic technologies: Simplifying goodness-of-fit methods using operations on technologies. Journal of Productivity Analysis, 14(3), 267-274.

Chambers, R.G., Chung, Y., Färe, R. (1998). Profit, directional distance functions, and Nerlovian efficiency. Journal of Optimization Theory and Applications, 98(2), 351-364.

Chambers, R.G., Färe, R. (2008). A "calculus" for data envelopment analysis. Journal of Productivity Analysis, 30(3), 169-175.

Charnes, A., Cooper, W.W., Rhodes, E. (1978). Measuring the efficiency of decision making units. European Journal of Operational Research, 2(6), 429-444.

Cherchye, L., De Rock, B., Dierynck, B., Roodhooft, F., Sabbe, J. (2013). Opening the "black box" of efficiency measurement: Input allocation in multioutput settings. Operations Research, 61(5), 1148-1165.

Cherchye, L., De Rock, B., Walheer, B. (2015). Multi-output efficiency with good and bad outputs. European Journal of Operational Research, 240(3), 872-881.

Cherchye, L., De Rock, B., Walheer, B (2016). Multi-output profit efficiency and directional distance functions. Omega, 61, 100-109. 
Cook, W.D., Zhu, J. (2011). Multiple variable proportionality in data envelopment analysis. Operations Research, 59(4), 1024-1032.

Cooper, W.W., Seiford, L.M., Tone, K. (2007). Data envelopment analysis. A comprehensive text with models, applications, references and DEA-Solver software (2nd ed.). New York: Springer Science + Business Media.

Deprins, D., Simar, L., Tulkens, H. (1984). Measuring labor-efficiency in post offices. In M. Marchand, P. Pestieau, H. Tulkens (Eds.), The performance of public enterprises: concepts and measurements (pp. 243267). Amsterdam: North-Holland.

Dyson, R.D., Thanassoulis, E. (1988). Reducing weight flexibility in data envelopment analysis. Journal of the Operational Research Society, 39(6), 563-576.

Färe, R., Grosskopf, S. (1985). A nonparametric cost approach to scale efficiency. Scandinavian Journal of Economics, 87(4), 594-604.

Färe, R., Grosskopf, S., Lovell, C.A.K. (1983). The structure of technical efficiency. Scandinavian Journal of Economics, 85(2), 181-190.

Färe, R., Grosskopf, S., Lovell, C.A.K. (1985). The measurement of efficiency of production. Boston: Kluwer Academic Publishers.

Førsund, F.R., Hjalmarsson, L. (2004). Calculating scale elasticity in DEA models. Journal of the Operational Research Society, 55(10), 1023-1038.

Fukuyama, H. (2000). Returns to scale and scale elasticity in data envelopment analysis. European Journal of Operational Research, 125(1), 93-112.

Hadjicostas, P., Soteriou, A.C. (2006). One-sided elasticities and technical efficiency in multi-output production: A theoretical framework. European Journal of Operational Research, 168(2), 425-449.

Hanoch, G. (1970). Homotheticity in joint production. Journal of Economic Theory, 2(4), 423-426.

Kerstens, K., Vanden Eeckaut, P. (1999). Estimating returns to scale using non-parametric deterministic technologies: A new method based on goodness-of-fit. European Journal of Operational Research, 113(1), $206-214$.

Joro, T., Korhonen, P.J. (2015). Extension of data envelopment analysis with preference information: Value efficiency. New York: Springer Science + Busines Media.

Kao, C. (2014). Network data envelopment analysis: A review. European Journal of Operational Research, $239(1), 1-16$.

Kuosmanen, T. (2005). Weak disposability in nonparametric productivity analysis with undesirable outputs. American Journal of Agricultural Economics, 87(4), 1077-1082.

Kuosmanen, T., Kazemi Matin, R. (2011). Duality of weakly disposable technology. Omega, 39(5), 504-512.

Kuosmanen, T., Podinovski, V.V. (2009). Weak disposability in nonparametric production analysis: Reply to Färe and Grosskopf. American Journal of Agricultural Economics, 91(2), 539-545.

Panzar, J.C., Willig, R.D. (1977). Economies of scale in multi-output production. The Quarterly Journal of Economics, 91(3), 481-491.

Podinovski, V.V. (2004a). Efficiency and returns to scale on the "no free lunch assumption only. Journal of Productivity Analysis, 22(3), 227-257.

Podinovski, V.V. (2004b). Local and global returns to scale in performance measurement. Journal of the Operational Research Society, 55(2), 170-178.

Podinovski, V.V. (2004c). Bridging the gap between the constant and variable returns-to-scale models: Selective proportionality in data envelopment analysis. Journal of the Operational Research Society, 55(3), 265-276.

Podinovski, V.V. (2004d). Production trade-offs and weight restrictions in data envelopment analysis. Journal of the Operational Research Society, 55(12), 1311-1322.

Podinovski, V.V. (2004e). On the linearisation of reference technologies for testing returns to scale in FDH models. European Journal of Operational Research, 152(3), 800-802.

Podinovski, V.V. (2005). The explicit role of weight bounds in models of data envelopment analysis. Journal of the Operational Research Society, 56(12), 1408-1418.

Podinovski, V.V. (2007). Improving data envelopment analysis by the use of production trade-offs. Journal of the Operational Research Society, 58(10), 1261-1270.

Podinovski, V.V. (2009). Production technologies based on combined proportionality assumptions. Journal of Productivity Analysis, 32(1), 21-26.

Podinovski, V.V. (2015). DEA models with production trade-offs and weight restrictions. In J. Zhu (Ed.), Data envelopment analysis: A handbook of models and methods (pp. 105-144). New York: Springer Science + Busines Media.

Podinovski, V.V. (2016). Optimal weights in DEA models with weight restrictions. European Journal of Operational Research, 254(3), 916-924. 
Podinovski, V.V., Bouzdine-Chameeva, T. (2011). The impossibility of convex constant returns-to-scale production technologies with exogenously fixed factors. European Journal of Operational Research, 213(1), 119-123.

Podinovski, V.V., Bouzdine-Chameeva, T. (2013). Weight restrictions and free production in data envelopment analysis. Operations Research, 61(2), 426-437.

Podinovski, V.V., Bouzdine-Chameeva, T. (2015). Consistent weight restrictions in data envelopment analysis. European Journal of Operational Research, 244(1), 201-209.

Podinovski, V.V., Chambers, R.G., Atici, K.B., Deineko, I.D. (2016). Marginal values and returns to scale for nonparametric production frontiers. Operations Research, 64(1), 236-250.

Podinovski, V.V., Førsund, F.R. (2010). Differential characteristics of efficient frontiers in data envelopment analysis. Operations Research, 58(6), 1743-1754.

Podinovski, V.V., Førsund, F.R., Krivonozhko, V.E. (2009). A simple derivation of scale elasticity in data envelopment analysis. European Journal of Operational Research, 197(1), 149-153.

Podinovski, V.V., Ismail, I., Bouzdine-Chameeva, T., Zhang, W. (2014). Combining the assumptions of variable and constant returns to scale in the efficiency evaluation of secondary schools. European Journal of Operational Research, 239(2), 504-513.

Ray, S.C. (2004). Data envelopment analysis. Theory and techniques for economics and operations research. Cambridge: Cambridge University Press.

Ray, S.C., Hu, X. (1977). On the technically efficient organization of an industry: A study of U.S. airlines. Journal of Productivity Analysis, 8(1), 5-18.

Rockafellar, R.T. (1970). Convex analysis. Princeton, NJ: Princeton University Press.

Sahoo, B.K., Tone, K. (2015). Scale elasticity in non-parametric DEA approach. In J. Zhu (Ed.), Data envelopment analysis: A handbook of models and methods (pp. 269-290). New York: Springer Science + Busines Media.

Sahoo, B.K., Zhu, J., Tone, K., Klemen, B.M. (2014). Decomposing technical efficiency and scale elasticity in two-stage network DEA. European Journal of Operational Research, 233(3), 584-594.

Seiford, L.M., Thrall, R.M. (1990). Recent developments in DEA: The mathematical programming approach to frontier analysis. Journal of Econometrics, 46(1-2), 7-38.

Starrett, D. A. (1977). Measuring returns to scale in the aggregate, and the scale effect of public goods. Econometrica, 45(6), 1439-1455.

Thanassoulis, E., Portela, M.C., Allen, R. (2004). Incorporating value judgements in DEA. In: Cooper WW, Seiford LM, Zhu J, eds. In W.W. Cooper, L.M, Seiford, J. Zhu (Eds.), Handbook on data envelopment analysis (pp. 99-138). Boston: Kluwer Academic Publishers.

Thanassoulis, E., Portela, M.C.S., Despić, O. (2008). Data envelopment analysis: The mathematical programming approach to efficiency analysis. In H.O. Fried, C.A.K. Lovell, S.S. Schmidt (Eds.), The measurement of productive efficiency and productivity growth (pp. 251-420). New York: Oxford University Press.

Tone, K. (2001). On returns to scale under weight restrictions in data envelopment analysis. Journal of Productivity Analysis, 16(1), 31-47.

Zelenyuk, V. (2013). A scale elasticity measure for directional distance function and its dual: Theory and DEA estimation. European Journal of Operational Research, 228(3), 592-600. 